In honor of Roger Howe as a septuagenarian, and

in memory of Paul Sally Jr. and Joseph Shalika

\title{
COMPUTATIONS WITH BERNSTEIN PROJECTORS OF SL(2)
}

\author{
ALLEN MOY
}

\begin{abstract}
For the p-adic group $G=\mathrm{SL}(2)$, we present results of the computations of the sums of the Bernstein projectors of a given depth. Motivation for the computations is based on a conversation with Roger Howe in August 2013. The computations are elementary, but they provide an expansion of the delta distribution $\delta_{1_{G}}$ into an infinite sum of $G$-invariant locally integrable essentially compact distributions supported on the set of topologically unipotent elements. When these distributions are transferred, by the exponential map, to the Lie algebra, they give $G$-invariant distributions supported on the set of topologically nilpotent elements, whose Fourier Transforms turn out to be characteristic functions of very natural $G$-domains. The computations in particular rely on the SL(2) discrete series character tables computed by Sally-Shalika in 1968. This new phenomenon for general rank has also been independently noticed in recent work of Bezrukavnikov, Kazhdan, and Varshavsky.
\end{abstract}

\section{INTRODUCTION}

A key tool in harmonic analysis on a Lie group $G$ is the exponential map exp : $\mathfrak{g} \longrightarrow$ $G$ from the Lie algebra $\mathfrak{g}$ to the group $G$. The map, defined for all $X \in \mathfrak{g}$ is a local diffeomorphism of $0 \in \mathfrak{g}$ to $1 \in G$, and it is used to move functions and distributions, between $\mathfrak{g}$ and $G$, e.g., ones which are $G$-invariant and eigendistributions for the center of $\mathcal{U}(\mathfrak{g})$. When $F$ is a p-adic field, i.e., a local field of characteristic zero, and $G$ the $F$ rational points of a connected reductive group defined over $F$, and $\mathfrak{g}$ the Lie algebra of $G$, the exponential map is only defined on a certain $G$-invariant, open and closed subset containing $0 \in \mathfrak{g}$. In terms of Moy-Prasad filtrations [MPa,MPb], for $r \in \mathbb{R}$, set

$$
\mathfrak{g}_{r}:=\bigcup_{x \in \mathbb{B}} \mathfrak{g}_{x, r} \text { and } \mathfrak{g}_{r^{+}}:=\bigcup_{x \in \mathbb{B}, s>r} \mathfrak{g}_{x, s} .
$$

We recall these sets are $G$-domains, i.e., $G$-invariant open and closed subsets. The set $\mathfrak{g}_{0^{+}}$is the set of topologically nilpotent elements in $\mathfrak{g}$. The assumption $\operatorname{char}(F)=0$ means, there exists $R \geq 0$ so that for $r>R$, the exponential map exp is defined on the $G$-domain $\mathfrak{g}_{r}$ and for any $x \in \mathscr{B}$, exp takes $\mathfrak{g}_{x, r}$ bijectively to the Moy-Prasad group $G_{x, r}$, and $\mathfrak{g}_{r}$ bijectively to $G_{r}$. In the best situation $R$ is 0 .

We recall the two realizations $[\mathrm{B}]$ of the Bernstein center $\mathcal{Z}(G)$ :

1991 Mathematics Subject Classification. Primary 22E50, 22E35.

Key words and phrases. Bernstein center, Bernstein projector, depth, Fourier transform, Moy-Prasad filtrations, p-adic group Steinberg representation, topologically unipotent.

The author is partly supported by Hong Kong Research Grants Council grant CERG \#603813. 
- The geometrical realization. $\mathcal{Z}(G)$ is the algebra of $G$-invariant essentially compact distributions on $G$ - a distribution is essentially compact if $\forall f \in C_{0}^{\infty}(G)$, the convolutions $D \star f$ and $f \star D$ is in $C_{0}^{\infty}(G)$.

- The spectral realization. $\mathcal{Z}(G)$ is the product $\prod_{\Omega} \mathbb{C}(\Omega)$ over the Bernstein components $\Omega$ of the algebras of (complex) regular functions $\mathbb{C}(\Omega)$.

For a fixed Bernstein component $\Omega$, and a regular function $s \in \mathbb{C}(\Omega)$, it is known the distribution $s$ is representable by a locally integrable function supported, and locally constant on the regular set. Of particular interest is the idempotent distribution $e_{\Omega}$ whose spectral realization is the constant function 1 on $\Omega$. In the situation when $G$ is semisimple and the Bernstein $\Omega$ corresponds to an equivalence class of supercuspidal representations, then $e_{\Omega}$ is the distributional character of the class times its formal degree. In this setting $(G$ semisimple), an important result of Dat [D] states the distribution $e_{\Omega}$ is supported on the $G$-domain $\mathcal{C}$ of compact elements, i.e., elements which belong to a compact subgroup of $G$. We note the set of topologically unipotent elements $\mathcal{U}^{\text {top }}$ is contained in $\mathcal{C}$.

Based on known examples, e.g., for $\mathrm{SL}(2)$ see $[\mathrm{MT} 1, \mathrm{MTb}]$, if $s \in \mathbb{C}(\Omega)$ is viewed as a distribution, its support is generally not contained in $\mathcal{U}^{\text {top }}$. An intriguing question is whether it is possible to find an element in the span of finitely many $\mathbb{C}(\Omega)$ whose support is contained in $\mathcal{U}^{\text {top }}$.

In $[\mathrm{MPa}, \mathrm{MPb}]$, the depth invariant $\rho(\pi)$ is defined for any irreducible smooth representation $\pi$, and it is known the depth is the same for all irreducible representation (classes) occurring in a Bernstein component. Thus, a depth $\rho(\Omega)$ is attached to any Bernstein component $\Omega$.

Let $d \geq 0$ be the depth of a Bernstein component, and set

$$
e_{d}:=\sum_{\rho(\Omega)=d} e_{\Omega} \text { and } \sigma_{d}:=\sum_{\rho(\Omega) \leq d} e_{\Omega} .
$$

Here we show, for $G=\operatorname{SL}(2, F)$, the Bernstein center element $\sigma_{d}$ has support in the topological unipotent set

$$
\mathcal{U}_{d^{+}}^{\text {top }}=\bigcup_{x \in \mathscr{B}} G_{x, d^{+}}
$$

Recall (i) an element $y \in G$ is called split (resp. elliptic), if its characteristic polynomial has distinct roots in $F$ (resp. not in $F$ ), and (ii) the depth of an irreducible representations is a half-integer, i.e, in $\frac{1}{2} \mathbb{N}$.

Theorem. 8.2. Suppose $F$ is a p-adic field with odd residue characteristic, and $G=$ $\mathrm{SL}(2, F)$. For $d \in \frac{1}{2} \mathbb{N}$, set $d^{+}:=k+\frac{1}{2}$. Then, we have $\operatorname{supp}\left(\sigma_{d}\right) \subset \mathcal{U}_{d^{+}}^{\text {top }}$, and on $\mathcal{U}_{d^{+}}^{\text {top }}$ :

- When d is integral:

$$
\sigma_{d}(y)=\left(q^{2}-1\right) q^{3 d}\left\{\begin{array}{l}
\left(\frac{2 q^{-d}}{\left|\alpha-\alpha^{-1}\right|_{F}}-1\right) \text { when } y \text { is split with eigenvalues } \alpha, \alpha^{-1} \\
-1 \quad \text { when } y \text { is elliptic }
\end{array}\right.
$$


- When d is half-integral:

$\sigma_{d}(y)=\left(q^{2}-1\right) q^{3 d+\frac{1}{2}}\left\{\begin{array}{l}\left(\frac{2 q^{-\left(d+\frac{1}{2}\right)}}{\left|\alpha-\alpha^{-1}\right|_{F}}-1\right) \quad \text { when } y \text { is split with eigenvalues } \alpha, \alpha^{-1} \\ -1 \quad \text { when } y \text { is elliptic }\end{array}\right.$

We observe, for $\mathrm{SL}(2)$, the projector $\sigma_{0}$ is equal to the Steinberg character restricted to the topological unipotent set.

As mentioned above, there is an $R \geq 0$ so that the exponential power series $\exp (X)$ is convergent when the eigenvalues of $X$ have normalize valuations greater than $R$, and for $r>R$, the exponential map is then a bijection between the set $\mathcal{N}_{r}^{\text {top }} \subset \mathfrak{g}$, and the set $\mathcal{U}_{r}^{\text {top }} \subset G$. In this ideal situation, we can then move the distributions in (8.2) to the Lie algebra. For $d \in \frac{1}{2} \mathbb{N}$, satisfying $d>R$, the Lie algebra distribution $\sigma_{d} \circ$ exp has support in $\mathfrak{g}_{d^{+}}$, and we have the homogeneity relation

$$
\left(\sigma_{d+1} \circ \exp \right)(\varpi Y)=q^{3}\left(\sigma_{d} \circ \exp \right)(Y) \text {. }
$$

Whence, their Fourier transforms satisfy the homogeneity relation

$$
\operatorname{FT}\left(\sigma_{k+1} \circ \exp \right)\left(\varpi^{-1} Y\right)=\operatorname{FT}\left(\sigma_{k} \circ \exp \right)(Y)
$$

In this regard, we show in the appendix the following:

Proposition. A.1. For $\mathfrak{s l}(2)$, we have

- The Fourier transforms $F T\left(1_{\mathfrak{g}_{0}}\right)$ and $F T\left(1_{\mathfrak{g}_{-\frac{1}{2}}}\right)$ have support in the sets $\mathfrak{g}_{0^{+}}:=\mathfrak{g}_{\frac{1}{2}}$ and $\mathfrak{g}_{\left(\frac{1}{2}\right)^{+}}:=\mathfrak{g}_{1}$ respectively.

In particular, the support is contained in $\mathcal{N}^{\text {top }}$.

- For $k \geq 1$, the Fourier transform $F T\left(1_{\mathfrak{g}_{-k}}\right)$ has support in $\mathfrak{g}_{k^{+}}:=\mathfrak{g}_{k+\frac{1}{2}}$.

For a general connected reductive p-adic group, when $R=0$ and other conditions, Kim $[\mathrm{Ka}, \mathrm{Kb}]$, showed, for $X$ in $\mathfrak{g}_{\left(\frac{d}{2}\right)^{+}}$:

$$
\int_{\widehat{G}_{\leq d}^{\mathrm{temp}}} \Theta_{\pi}(\exp (X)) d \mu_{\mathrm{PM}}(\pi)=F T\left(1_{\mathfrak{g}_{-d}}\right)(X) .
$$

The integral is over the (classes of) irreducible tempered representations of depth less than or equal to $d$. Thus, for $\mathrm{SL}(2)$, when $R=0$ we have

$$
\sigma_{d} \circ \exp =F T\left(1_{\mathfrak{g}_{-d}}\right) \quad\left(\text { both sides have support in } \mathfrak{g}_{d^{+}}\right) \text {. }
$$

We conjecture, for SL(2), and more generally for any connected reductive p-adic group, this identity to be true even when $R>0$, as long as $d>R$.

In October 2014, through correspondence with Roman Bezrukavnikov, the author became aware of unpublished work in-progress of Bezrukavnikov, Kazhdan, and Varshavsky in which 
they independently discovered and proved, for a general connected reductive p-adic group, the support of the projector $\sigma_{d}$ is in the topological unipotent set, that $\sigma_{0}$ is the restriction of the Steinberg character to the unipotent set, and the identification of the Fourier transforms $\mathrm{FT}\left(\sigma_{d} \circ \exp \right)$. A preprint [BKV] of their work became available in April 2015.

Motivation for considering the support of the depth zero projector $e_{0}$, the sum of the projectors $e_{\Omega}$ with $\rho(\Omega)=0$, aroused during a conversation the author had with Roger Howe in August 2013, and the author successfully verified the support is contained in $\mathcal{U}_{0^{+}}^{\text {top }}$ and a formula for the values in December 2013. Extension of the support and values of $\sigma_{d}$ to all depths was completed in March 2015 while the author was a visiting faculty at the University of Utah. The author kindly thanks the hospitality of the Mathematics Department of the University of Utah, with special thanks to Dragan Miličić. The author gratefully acknowledges useful conversations with Roman Bezrukavnikov, Roger Howe, Ju-Lee Kim, and Fiona Murnaghan. The author gave workshop talks of the case $e_{0}$ at the Mathematical Research Institute of Oberwolfach, the University of Zagreb, and the University of Chicago, and thanks these institutions for their invitations.

\section{Notation}

We set some notation. Let $F$ denote a p-adic field (so of characteristic zero) . Let $\mathcal{R}_{F}$ denote the ring of integers of $F$, let $\wp_{F}$ its prime ideal, and let $\varpi$ be a prime element. Set $\mathbb{F}_{q}=\mathcal{R}_{F} / \wp_{F}$ to be the residue field. To be able to use the Sally-Shalika character tables [SS], we assume the residue characteristic of $F$ is odd.

Let $\mathcal{B}$ be the Bruhat-Tits building $G=\operatorname{SL}(2, F)$. The group $\operatorname{GL}(2, F)$, whence also $G$, acts on $\mathcal{B}$. There are respectively two $G$-orbits, and one $\mathrm{GL}(2, F)$-orbit of vertices in $\mathcal{B}$. The maximal compact subgroups of $G$ are precisely the stabilizer subgroups $G_{x}$ (in $G$ ) of vertices $x$ in $\mathcal{B}$; whence, there two conjugacy classes of maximal compact subgroups in $G$. Let $x_{0}$ and $x_{1}$ be the vertices in $\mathcal{B}$ so that

$$
G_{x_{0}}=\operatorname{SL}\left(2, \mathcal{R}_{F}\right), \text { and } G_{x_{1}}=\left[\begin{array}{cc}
\varpi^{-1} & 0 \\
0 & 1
\end{array}\right] \operatorname{SL}\left(2, \mathcal{R}_{F}\right)\left[\begin{array}{cc}
\varpi & 0 \\
0 & 1
\end{array}\right]
$$

are the familiar representatives of the two conjugacy classes of maximal compact subgroups of $G$. If $e$ is an edge (with vertices $y$ and $z$ and (midpoint) barycenter $b(e)$ ) in $\mathcal{B}$, then the stabilizer (in $G$ ) of $e$ is an Iwahori subgroup equal to $G_{y} \cap G_{z}=G_{b(e)}$.

We note the two vertices $x_{0}$ and $x_{1}$ mentioned in (2.1) are the vertices of an edge $e_{01} \in \mathcal{B}$. Let $x_{01}=b\left(e_{01}\right)$ - the barycenter of $e_{01}$. The Iwahori subgroup $G_{x_{01}}$ equals:

$$
G_{x_{01}}=G_{x_{0}} \cap G_{x_{1}}=\left\{g \in \mathrm{SL}\left(2, \mathcal{R}_{F}\right) \mid g \text { upper triangular } \bmod \wp_{F}\right\} .
$$

For notational convenience, we set 


$$
\mathcal{K}=G_{x_{0}}, \quad \mathcal{K}^{\prime}=G_{x_{1}}, \quad \mathcal{I}=\mathcal{K} \cap \mathcal{K}^{\prime}=G_{x_{01}}
$$

Set $\mathfrak{g}=\mathfrak{s l}(2, F)$. For any $x \in \mathcal{B}$, let

$$
G_{x, r} \text { and } r \geq 0 \quad \text { and } \quad \mathfrak{g}_{x, r} \text { and } r \in \mathbb{R}
$$

be the Moy-Prasad filtration subgroups. We recall:

(i) If $x \in \mathcal{B}$ is a vertex, then the jumps in the filtration subgroups $G_{x, r}$ occur at integral $r$, i.e.,

$$
G_{x, r^{+}}=G_{x, r+1} \quad \text { when } \quad r \in \mathbb{N}
$$

(ii) If $x=b(e)$ is the barycenter of an edge $e$, then the jumps in the filtration subgroups occur at half-integral $r$, i.e.,

$$
G_{x, r^{+}}=G_{x, r+\frac{1}{2}} \quad \text { when } \quad r \in \frac{1}{2} \mathbb{N}
$$

and similarly for $\mathfrak{g}_{x, r}$. For the latter, we have

$$
\mathfrak{g}_{x, r+1}=\varpi \mathfrak{g}_{x, r}
$$

Take $\psi$ to be an additive character of $F$ which has conductor $\wp_{F}$.

(iii) For $2 r \geq s$, the quotient group $G_{x, r} / G_{x, s}$ is abelian and canonically isomorphic to $\mathfrak{g}_{x, r} / \mathfrak{g}_{x, s}$. The residual characteristic is odd assumption means the trace pairing

$$
\mathfrak{g}_{x, r} / \mathfrak{g}_{x, s} \times \mathfrak{g}_{x,-s^{+}} / \mathfrak{g}_{x,-r^{+}} \rightarrow F / \wp_{F}
$$

allows us to identify the Pontryagin dual $\left(\mathfrak{g}_{x, r} / \mathfrak{g}_{x, s}\right)^{\wedge}\left(\right.$ whence $\left(G_{x, r} / G_{x, s}\right)^{\wedge}$ with $\mathfrak{g}_{x,-s^{+}} / \mathfrak{g}_{x,-r^{+}}$. A coset $\Xi=X+\mathfrak{g}_{x,-r^{+}}$yields the character $\psi_{\Xi}$ of $\mathfrak{g}_{x, r} / \mathfrak{g}_{x, s}$ given as:

$$
\psi_{\Xi}(Y):=\psi(\operatorname{trace}(X Y)) .
$$

We note:

- For the vertex $x_{0}$ :

$$
\mathfrak{g}_{x_{0}, 0} / \mathfrak{g}_{x_{0}, 1}=\mathfrak{s l}\left(2, \mathbb{F}_{q}\right) .
$$

For a general vertex $x \in \mathcal{B}$ a vertex, and $r \in \mathbb{N}$ :

$$
\left(\mathfrak{g}_{x, r} / \mathfrak{g}_{x,(r+1)}\right)^{-}=\mathfrak{g}_{x,-r} / \mathfrak{g}_{x,(-r+1)} \simeq \mathfrak{g}_{x, 0} / \mathfrak{g}_{x, 1} \simeq \mathfrak{s l}\left(2, \mathbb{F}_{q}\right)
$$

The isomorphism $\mathfrak{g}_{x,-r} / \mathfrak{g}_{x,(-r+1)} \simeq \mathfrak{g}_{x, 0} / \mathfrak{g}_{x, 1}$ is the natural one given by scalar multiplication by $\varpi$. The isomorphism $\mathfrak{g}_{x, 0} / \mathfrak{g}_{x, 1} \simeq \mathfrak{s l}\left(2, \mathbb{F}_{q}\right)$ is up to a conjugation by by an element of $\mathrm{GL}\left(2, \mathbb{F}_{q}\right)$. A coset $X \in \mathfrak{g}_{x,-r} / \mathfrak{g}_{x,(-r+1)}$ is, by definition, non-degenerate if, as an element in $\left.\mathfrak{s l}\left(2, \mathbb{F}_{q}\right)\right)$, it is non-nilpotent. 
- For the barycenter point $x_{01}$ :

$$
\mathfrak{g}_{x_{01}, \frac{1}{2}} / \mathfrak{g}_{x_{01}, 1}=\left\{\left[\begin{array}{cc}
\varpi a & b \\
\varpi c & -\varpi a
\end{array}\right] \mid a, b, c \in \mathcal{R}_{F}\right\} /\left\{\left[\begin{array}{cc}
\varpi a & \varpi b \\
\varpi^{2} c & -\varpi a
\end{array}\right] \mid a, b, c \in \mathcal{R}_{F}\right\} .
$$

For $d \in \mathbb{N}$ :

$$
\left(\mathfrak{g}_{x_{01}, d+\frac{1}{2}} / \mathfrak{g}_{x_{01},(d+1)}\right)^{-}=\mathfrak{g}_{x_{01},-d-\frac{1}{2}} / \mathfrak{g}_{x_{01},-d}
$$

We recall a coset

$$
X=\varpi^{-(d+1)}\left[\begin{array}{cc}
\varpi a & b \\
\varpi c & -\varpi a
\end{array}\right]+\mathfrak{g}_{x_{01},-d} \in \mathfrak{g}_{x_{01},-d-\frac{1}{2}} / \mathfrak{g}_{x_{01},-d} \quad\left(a, b, c \in \mathcal{R}_{F}\right)
$$

is non-degenerate if $b$ and $c$ are both units.

\section{REVIEW OF EARLIER RESULtS}

3.1. A result of J. Dat on support. Suppose $G$ is a general connected reductive p-adic group. A compact element $\gamma \in G$ is one which lies in a compact subgroup of $G$. Set

$$
\mathcal{C}:=\text { set of compact elements. }
$$

The following important result of Dat says the support of a projector $e_{\Omega}$ to a Bernstein component $\Omega$ is contained in $\mathcal{C}$.

Theorem 3.1.1. (Dat, $[\mathrm{D}])$ Suppose $G$ is a connected reductive $p$-adic group. Let $\mathcal{C}$ be the set of compact elements in $G$. For any Bernstein component $\Omega$, let $e_{\Omega}$ be the element of the Bernstein center which projects onto the component $\Omega$. Then

$$
\operatorname{supp}\left(e_{\Omega}\right) \subset \mathcal{C} .
$$

3.2. A result of Moy-Prasad on depths. If $\pi$ is an irreducible smooth representation of a connected reductive p-adic group, let $\rho(\pi)$ denote its depth as defined in [MPa, $\mathrm{MPb}$. We recall the following result on depths of representations which implies we can define the depth of a Bernstein component.

Theorem 3.2.1. (Moy-Prasad, [MPb], Theorem 5.2) Suppose $G$ is a connected reductive $p$-adic group, $M N$ is a parabolic subgroup, and $\sigma$ an irreducible smooth representation of $M$. If $\pi$ is any irreducible subquotient of $\operatorname{Ind}_{M N}^{G}(\sigma)$, then $\rho(\pi)=\rho(\sigma)$.

Let $d \geq 0$ be the depth of a Bernstein component, and set

$$
e_{d}:=\sum_{\rho(\Omega)=d} e_{\Omega} \text { and } \sigma_{d}:=\sum_{\rho(\Omega) \leq d} e_{\Omega} .
$$

As mentioned in the introduction, when $G=\mathrm{SL}(2)$, in the remainder of this manuscript, we show $\sigma_{d}$ has support in the topological unipotent set $\mathcal{U}^{\text {top }}$, and indeed in the smaller $G$-domain $\mathcal{U}_{d^{+}}^{\text {top }}$ 
3.3. A partition of the compact elements $\mathcal{C}$ of $\mathrm{SL}(2)$. We partition $\mathcal{C}$ into three subsets:

$$
\mathcal{C}=\mathcal{U}^{\text {top }} \coprod-\mathrm{I}_{2 \times 2} \mathcal{U}^{\text {top }} \coprod \mathcal{C}_{\text {st-reg }}
$$

Here, $\mathcal{C}_{\text {st-reg }}$ is the set of 'strongly regular' elements, i.e., those elements whose eigenvalues are not congruent to each other modulo the prime.

\section{Principal Series projectors for SL(2)}

For SL(2), Moy-Tadić [MT1,MTb] explicitly computed the projectors $e_{\Omega}$ for principal series components. To state the results, we normalize Haar measure on SL(2) so that

$$
\operatorname{meas}\left(\operatorname{SL}\left(2, \mathcal{R}_{F}\right)\right)=1 \text {. }
$$

We enumeration the Principal Series Bernstein components as: (i) $\Omega\left(\left\{\chi, \chi^{-1}\right\}\right.$ ), where $\chi$ is a character of $\mathcal{R}_{F}^{\times}$with $\chi \neq \chi^{-1}$, (ii) $\Omega(\operatorname{sgn})$, with sgn the order two character of $\mathcal{R}_{F}^{\times}$, and (iii) $\Omega=\Omega_{\text {triv }}$, the Bernstein component of irreducible representations with non-zero Iwahori-fixed vectors. Let $f(\chi)$ denote the conductor of $\chi$. The Principal Series projectors are:

Regular PS $f(\chi)=d+1$

$$
e_{\Omega\left(\left\{\chi, \chi^{-1}\right\}\right)}(y)=\left\{\begin{array}{l}
(q+1) q^{d} \frac{\chi(\alpha)+\chi\left(\alpha^{-1}\right)}{\left|\alpha-\alpha^{-1}\right|_{F}}, \\
y \text { split with eigenvalues } \alpha, \alpha^{-1} \\
0 \quad \text { otherwise }
\end{array}\right.
$$

Sgn PS

$$
e_{\Omega(\operatorname{sgn})}(y)=\left\{\begin{array}{c}
(q+1) \frac{\operatorname{sgn}(\alpha)}{\left|\alpha-\alpha^{-1}\right|_{F}} \\
y \text { split with eigenvalues } \alpha, \alpha^{-1} \\
0 \quad \text { otherwise }
\end{array}\right.
$$

Unramified PS (Iwahori fixed vectors)

$$
e_{\Omega}(y)=\left\{\begin{array}{c}
\frac{2 q}{\left|\alpha-\alpha^{-1}\right|_{F}}-(q-1) \\
y \text { split with eigenvalues } \alpha, \alpha^{-1} \\
-(q-1) \quad y \text { elliptic }
\end{array}\right.
$$

Let $e_{d}^{\mathrm{PS}}$ be the sum of the principal series depth $d$ Bernstein projectors. 
For $d=0$ we have:

$$
e_{0}^{\mathrm{PS}}(y)=(q-1)\left\{\begin{aligned}
& \frac{(q+2)}{\left|\alpha-\alpha^{-1}\right|}-1 \text { when } y \in \mathcal{U}^{\text {top }} \text { is split with eigenvalues } \alpha, \alpha^{-1} \\
& \frac{1}{\left|\alpha-\alpha^{-1}\right|}-1 \text { when } y \in-\mathrm{I}_{2 \times 2} \mathcal{U}^{\text {top }} \text { is split with eigenvalues } \alpha, \alpha^{-1} \\
& 0 \quad \text { when } y \in \mathcal{C}_{\text {st-reg }} \text { is split } \\
&-1 \quad \text { when } y \in \mathcal{C} \text { is elliptic }
\end{aligned}\right.
$$

For $d>0$, each principal series Bernstein projector $e_{\Omega}$ has support in the split set and consequently the support of $e_{d}^{\mathrm{PS}}$ is in the split set too. For $y \in \mathrm{SL}(2)$ a (regular) split element, let $\alpha, \alpha^{-1}$ be the eigenvalues of $y$. We have:

$e_{d}^{\mathrm{PS}}(y)=(q+1) q^{d}(q-1) q^{d-1} \begin{cases}\frac{(-1)}{\left|\alpha-\alpha^{-1}\right|}=-q^{d} \quad \text { when } \alpha, \alpha^{-1} \text { in }\left(1+\wp_{F}^{d}\right) \backslash\left(1+\wp_{F}^{d+1}\right) \\ \frac{(q-1)}{\left|\alpha-\alpha^{-1}\right|} & \text { when } \alpha, \alpha^{-1} \text { in }\left(1+\wp_{F}^{d+1}\right) \\ 0 & \text { otherwise }\end{cases}$

5. THE PROJECTOR $e_{0}$

Set

$$
e_{0}^{\text {cusp }}=\sum_{\substack{\pi \text { cuspidal } \\ \text { depth } 0}} e_{\pi} .
$$

In this section, we determine, with the aid of the Sally-Shalika tables [SS], the values of $e_{0}^{\text {cusp }}$. To conveniently use their tables we use their normalization of Haar measure, i.e., $\operatorname{meas}\left(G_{x_{0}}\right)=1$, as in (4.1).

5.1. Irreducible cuspidal representations of depth zero. $\quad$ Let $\mathcal{K}=G_{x_{0}}$, and $\mathcal{K}^{\prime}=$ $G_{x_{1}}$. We recall $\mathcal{K} / G_{x_{0}, 1} \simeq \operatorname{SL}\left(2, \mathbb{F}_{q}\right) \simeq \mathcal{K}^{\prime} / G_{x_{1}, 1}$.

\section{Proposition 5.1.1.}

(i) If $\kappa$ is an irreducible cuspidal representation of $\mathrm{SL}\left(2, \mathbb{F}_{q}\right)$, let $\kappa_{x_{0}}$ and $\kappa_{x_{1}}$ denote its inflation to $\mathcal{K}=G_{x_{0}}$ and $\mathcal{K}^{\prime}=G_{x_{1}}$ respectively. Then, the representations $\mathrm{c}-\operatorname{Ind}_{\mathcal{K}}^{G}\left(\kappa_{x_{0}}\right)$ and $\mathrm{c}-\operatorname{Ind}_{\mathcal{K}^{\prime}}^{G}\left(\kappa_{x_{1}}\right)$ are irreducible supercuspidal representations of $G$. Furthermore, the supercuspidal representations induced from the group $\mathcal{K}$ are inequivalent to those induced from the group $\mathcal{K}^{\prime}$. 
(ii) Any irreducible supercuspidal representation $\left(\pi, V_{\pi}\right)$ of depth zero is equivalent to a $\mathrm{c}-\operatorname{Ind}_{\mathcal{K}}^{G}\left(\kappa_{x_{0}}\right)$ or $a \mathrm{c}-\operatorname{Ind}_{\mathcal{K}^{\prime}}^{G}\left(\kappa_{x_{1}}\right)$

(iii) Normalize Haar measure on $\mathrm{SL}(2)$ so meas $(\mathcal{K})=1$. If $\kappa$ is a cuspidal representation of $\operatorname{SL}\left(2, \mathbb{F}_{q}\right)$ inflated to $\mathcal{K}$, and $\pi=\mathrm{c}_{-} \operatorname{Ind}_{\mathcal{K}}^{G}(\kappa)$, then

the formal degree $d_{\pi}=\operatorname{degree}(\kappa)$.

Similarly for $\mathcal{K}^{\prime}$.

We briefly review the cuspidal representations of $\operatorname{SL}\left(2, \mathbb{F}_{q}\right)$ :

- Let $T \subset \operatorname{SL}\left(2, \mathbb{F}_{q}\right)$ be the (elliptic) torus of order $(q+1)$. Take $\beta$ to be a primitive root of $T$, set $\zeta=e^{\frac{2 \pi \sqrt{-1}}{(q+1)}}$, and for integral $0 \leq i \leq q$, let $\theta_{i} \in \widehat{T}$ be the character $\theta_{i}(\beta)=\zeta^{i}$. For $0<i$, the character $\theta_{i}$ is conjugate under the normalizer $N_{G}(T)$ to $\theta_{(q+1)-i}$.

- For $i=1,2, \ldots, q$, there is a cuspidal representation $\sigma_{i}$ of $\operatorname{SL}\left(2, \mathbb{F}_{q}\right)$ of dimension $(q-1)$. Let $\chi_{i}$ be the character of $\sigma_{i}$. Then, $\chi_{i}=\chi_{(q+1)-i}$. For $i \neq \frac{(q+1)}{2}$, the character is irreducible, and for $i=\frac{(q+1)}{2}$, the character is the sum of two irreducible characters $\eta_{1}, \eta_{2}$ of degree $\frac{q-1}{2}$. Let $\epsilon \in \mathbb{F}_{q}^{\times}$be a non-square. In all cases, the support of $\chi_{i}$ is on the elements:

$$
\mathrm{Id}, \quad-\mathrm{Id}, \quad \beta^{k}, \quad u_{1}=\left[\begin{array}{ll}
1 & 1 \\
0 & 1
\end{array}\right], \quad u_{\epsilon}=\left[\begin{array}{ll}
1 & \epsilon \\
0 & 1
\end{array}\right], \quad-u_{1}, \quad-u_{\epsilon}
$$

with values:

\begin{tabular}{|c|c|c|c|c|c|c|c|}
\hline & Id & $-I d$ & $\beta^{k}$ & $u_{1}$ & $u_{\epsilon}$ & $-u_{1}$ & $-u_{\epsilon}$ \\
\hline$\chi_{i}$ & $(q-1)$ & $(-1)^{i}(q-1)$ & $-\left(\zeta^{i k}+\zeta^{-i k}\right)$ & -1 & -1 & $(-1)^{(i+1)}$ & $(-1)^{(i+1)}$ \\
\hline
\end{tabular}

Set $\pi_{i}:=\mathrm{c}-\operatorname{Ind}_{\mathcal{K}}^{G}\left(\sigma_{i}\right)$, and let $\Theta_{i}$ be the character of $\pi_{i}$. Note that $\pi_{i}(-\mathrm{Id})$ is $(-1)^{i}$ times the identify operator. If we use $\mathcal{K}^{\prime}$ instead of $\mathcal{K}$, we can define analogous representations $\pi_{i}^{\prime}$ and characters $\Theta_{i}^{\prime}$. Set

$$
\begin{aligned}
& e_{\mathcal{K}}:=(q-1) \sum_{1 \leq i \leq \frac{(q-1)}{2}} \Theta_{i}+\frac{(q-1)}{2} \Theta_{\left(\frac{(q+1)}{2}\right)}=\frac{(q-1)}{2} \sum_{1 \leq i \leq q} \Theta_{i} \\
& e_{\mathcal{K}^{\prime}}:=(q-1) \sum_{1 \leq i \leq \frac{(q-1)}{2}} \Theta_{i}^{\prime}+\frac{(q-1)}{2} \Theta_{\left(\frac{(q+1)}{2}\right)}^{\prime}=\frac{(q-1)}{2} \sum_{1 \leq i \leq q} \Theta_{i}^{\prime},
\end{aligned}
$$

so $e_{0}=e_{0}^{\mathrm{PS}}+e_{\mathcal{K}}+e_{\mathcal{K}^{\prime}}$. 
5.2. $e_{0}$ on split regular elements. Suppose $y \in G$ is a regular split compact element with eigenvalues $\alpha, \alpha^{-1}$. From Tables 2 and 3 (pages 1235-1236) of Sally-Shalika [SS]:

$$
\Theta_{i}(y)= \begin{cases}0 & \text { when } \alpha \neq \alpha^{-1} \bmod \wp \\ \frac{1}{\left|\alpha-\alpha^{-1}\right|}-1 & \text { when } \alpha \equiv 1 \bmod \wp\end{cases}
$$

Similarly for the character $\Theta_{i}^{\prime}$. We deduce

$$
e_{\mathcal{K}}(y)=\frac{(q-1)}{2} \begin{cases}0 & \text { when } \alpha \neq \alpha^{-1} \bmod \wp \\ (-1)\left(\frac{1}{\left|\alpha-\alpha^{-1}\right|}-1\right) & \text { when } \alpha \equiv-1 \bmod \wp \\ q\left(\frac{1}{\left|\alpha-\alpha^{-1}\right|}-1\right) & \text { when } \alpha \equiv 1 \bmod \wp\end{cases}
$$

and whence

$$
e_{0}(y)=(q-1)(q+1) \begin{cases}0 & \text { unless } \alpha \equiv 1 \bmod \wp \\ \left(\frac{2}{\left|\alpha-\alpha^{-1}\right|}-1\right) & \text { when } \alpha \equiv 1 \bmod \wp .\end{cases}
$$

5.3. $e_{0}$ on ramified elliptic elements. Suppose $y \in G$ is a ramified elliptic element, i.e., its eigenvalues $\alpha, \alpha^{-1}$ belong to a ramified quadratic extension $E$. We note either $y$ or $-y$ is topologically unipotent. We assume $y$ topologically unipotent, i.e., $\alpha \equiv 1 \bmod \wp_{E}$. From Table 2 (page 1235) of Sally-Shalika [SS]:

$$
\Theta_{i}(y)=-1, \text { therefore } \Theta_{i}(-y)=-1(-1)^{i} \text {, }
$$

and so

$$
e_{0}^{\text {cusp }}(y)=-(q-1) q \quad \text { and } \quad e_{0}^{\text {cusp }}(-y)=(q-1)
$$

We deduce

$$
\frac{1}{(q-1)(q+1)} e_{0}(y)= \begin{cases}0 & \text { when } y \text { is not topologically unipotent } \\ -1 & \text { when } y \text { is topologically unipotent }\end{cases}
$$


5.4. $e_{0}$ on unramified elliptic elements. Suppose $y \in G$ is a unramified elliptic element, i.e., its eigenvalues $\alpha, \alpha^{-1}$ belong to a unramified quadratic extension $E$. We consider the two cases depending on whether the two eigenvalues are congruent modulo $\wp_{E}$.

Case $\alpha \not \equiv \alpha^{-1} \bmod \wp_{E}:$ We note there are two $G$-conjugacy classes of elements which have eigenvalues $\alpha, \alpha^{-1}$. One class has non-empty intersection with $\mathcal{K}$, and empty intersection with $\mathcal{K}^{\prime}$, and vice versa for the other class. We assume $y \in \mathcal{K}$, and find, using Harish-Chandra's formula $[\mathrm{HC}]$ for the character of the compactly induced representation:

$$
e_{\mathcal{K}}(y)=\frac{(q-1)}{2} c-\operatorname{Ind}_{\mathcal{K}}^{G}\left(\sum_{1 \leq i \leq q} \Theta_{\sigma_{i}}\right)(y)=(q-1), \text { and } e_{\mathcal{K}^{\prime}}(y)=0
$$

If $y \in \mathcal{K}^{\prime}$, we have the obvious analogous situation. Therefore,

$$
e_{0}(y)=\left(e_{0}^{\mathrm{PS}}+e_{\mathcal{K}}+e_{\mathcal{K}^{\prime}}\right)(y)=(q-1)-(q-1)=0 \quad \text { when } \alpha \not \equiv \alpha^{-1} \bmod \wp_{E}
$$

Case $\alpha \equiv \alpha^{-1} \bmod \wp_{E}: \quad$ Here $\alpha \equiv \pm 1 \bmod \wp_{E}$. We assume $\alpha \equiv 1$, so $y$ is topologically unipotent. Let $\operatorname{sgn}_{E}$ denote the class-field character of $F^{\times}$of the unramified quadratic extension $E$. By Table 2 of Sally-Shalika [SS], the values of $\Theta_{i}(y)$ and $\Theta_{i}^{\prime}(y)$ in some order are:

$$
-1 \pm \operatorname{sgn}_{E}(\gamma) \frac{1}{\left|\alpha-\alpha^{-1}\right|}
$$

Here $\gamma= \pm \alpha-\alpha^{-1}$ is the (imaginary) part of $\alpha$. Note $\operatorname{sgn}_{E}( \pm 1)=1$. So,

$$
\left(\Theta_{i}+\Theta_{i}^{\prime}\right)(y)=2 \text { and }\left(\Theta_{i}+\Theta_{i}^{\prime}\right)(-y)=(-1)^{i} 2
$$

It follows:

$$
\begin{aligned}
\left(e_{\mathcal{K}}+e_{\mathcal{K}^{\prime}}\right)(y) & =\frac{(q-1)}{2} q(-2)=-(q-1) q, \text { and } \\
\left(e_{\mathcal{K}}+e_{\mathcal{K}^{\prime}}\right)(-y) & =\frac{(q-1)}{2}(-1)(-2)=(q-1) .
\end{aligned}
$$

Thus,

$$
e_{0}(y)=-(q-1)(q+1), \text { and } e_{0}(-y)=0 \quad \text { when } \alpha \equiv 1 \bmod \wp_{E} \text {. }
$$




\subsection{Summary Table of $e_{0}$.}

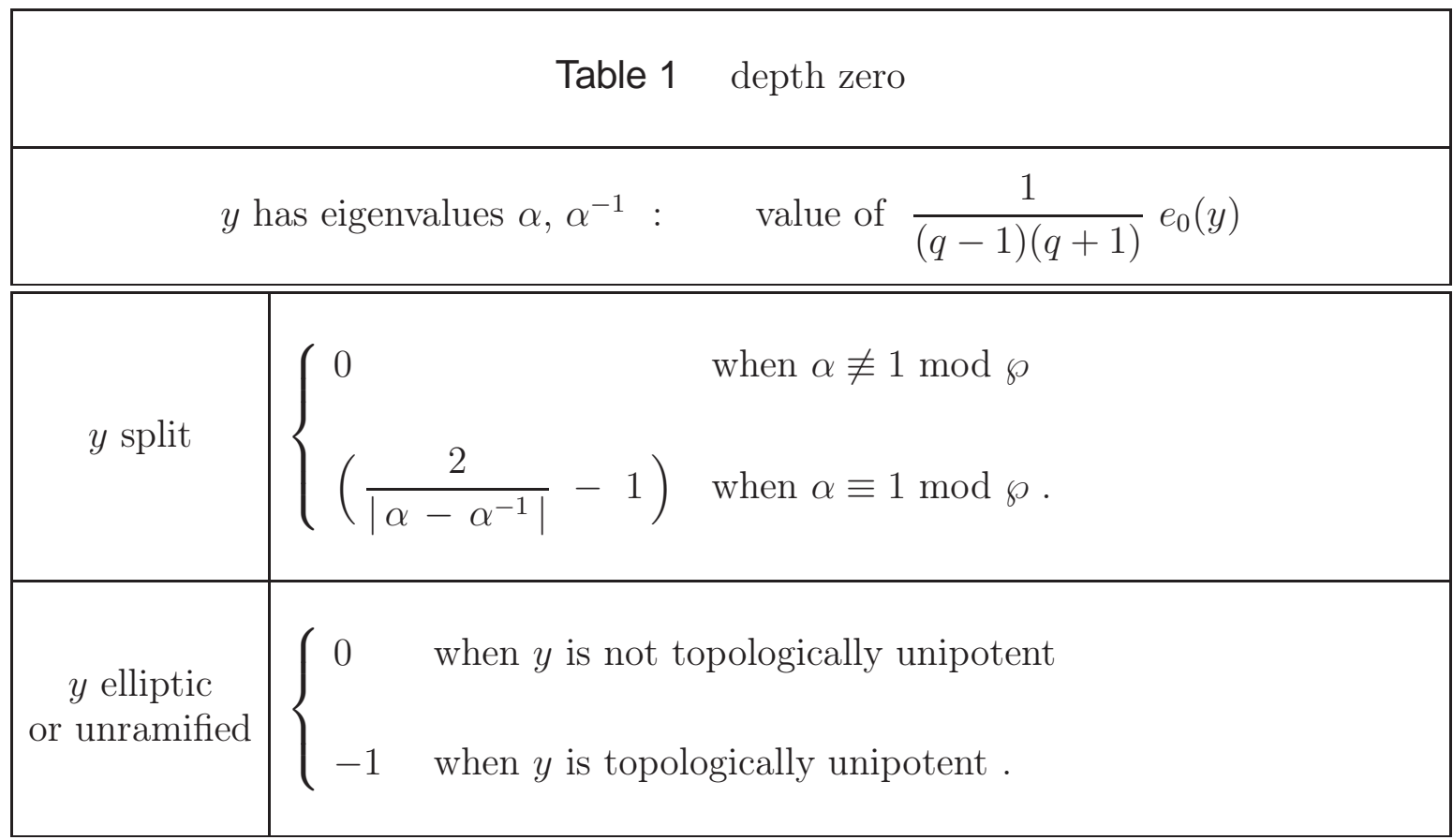

\section{INTEGRAL DEPTH SUPERCUSPIDAL REPRESENTATIONS}

6.1. The partition of supercuspidal representations by the groups $\mathcal{K}$ and $\mathcal{K}^{\prime}$. We abbreviate the filtration subgroups $\mathcal{K}=G_{x_{0}, r}$ as $\mathcal{K}_{r}(r \in \mathbb{N})$, and similarly we abbreviate the filtration subgroups of $\mathcal{K}^{\prime}=G_{x_{1}}$.

Suppose $d$ is a positive integer. We recall if $\left(\pi, V_{\pi}\right)$ is an irreducible supercuspidal representation $\left(\pi, V_{\pi}\right)$ of depth $d$, then either

$$
V_{\pi}^{\mathcal{K}_{d+1}} \neq\{0\} \quad, \quad \text { (exclusive) or } \quad V_{\pi}^{\mathcal{K}_{d+1}^{\prime}} \neq\{0\}
$$

In what follows, we assume $V_{\pi}^{\mathcal{K}_{d+1}} \neq\{0\}$ and remark the obvious transposition of results holds when $V_{\pi}^{\mathcal{K}_{d+1}^{\prime}} \neq\{0\}$.

The subgroup $\mathcal{K}_{d+1}$ is normal in $\mathcal{K}$, and therefore $V_{\pi}^{\mathcal{K}_{d+1}}$ is $\mathcal{K}$-invariant, and so $\mathcal{K}_{d}$-invariant. We note $\left(\mathcal{K}_{d} / \mathcal{K}_{d+1}\right)^{\wedge}=\mathfrak{g}_{x_{0},-d} / \mathfrak{g}_{x_{0},(-d+1)} \simeq \mathfrak{s l}\left(2, \mathbb{F}_{q}\right)$, and we recall:

(i) If $\left(\pi, V_{\pi}\right)$ is an irreducible supercuspidal representation of depth $d$ with $V_{\pi}^{\mathcal{K}_{d+1}} \neq\{0\}$, then the characters of $\mathcal{K}_{d} / \mathcal{K}_{d+1}$ which appear $V_{\pi}^{\mathcal{K}_{d+1}}$ have the form $\phi_{\Xi}$ where the coset $\Xi \in \mathfrak{g}_{x_{0},-d} / \mathfrak{g}_{x_{0},(-d+1)} \simeq \mathfrak{s l}\left(2, \mathbb{F}_{q}\right)$ is an elliptic element in $\mathfrak{s l}\left(2, \mathbb{F}_{q}\right)$, i.e., has irreducible characteristic polynomial. By Clifford theory, the set of $\phi_{\Xi}$ 's which appear in $V_{\pi}^{\mathcal{K}_{d+1}}$ form a single $\mathcal{K}$-orbit in $\mathfrak{g}_{x_{0},-d} / \mathfrak{g}_{x_{0},(-d+1)}$. 
(ii) The Adjoint action of $\mathcal{K}$ on $\mathfrak{s l}\left(2, \mathbb{F}_{q}\right)$ has $\frac{(q-1)}{2}$ orbits of elliptic elements, and each orbit contains $(q-1) q$ elliptic elements.

(iii) If $\phi_{\Xi}$ is a character of $\mathcal{K}_{d} / \mathcal{K}_{d+1}$ attached to an elliptic coset of $\Xi \in \mathfrak{g}_{x_{0},-d} / \mathfrak{g}_{x_{0},(-d+1)} \simeq$ $\mathfrak{s l}\left(2, \mathbb{F}_{q}\right)$, then

$$
c-\operatorname{Ind}_{\mathcal{K}_{d}}^{G}\left(\phi_{\Xi}\right)
$$

is a finite length (completely reducible) supercupsidal representation. If $\left(\pi, V_{\pi}\right)$ is as in part (i), i.e., $V_{\pi}^{\mathcal{I}_{d^{+}}}$contains the (non-degenerate) character $\phi_{\Xi}$, then by Frobenius reciprocity:

Furthermore:

$$
\operatorname{Hom}_{G}\left(V_{\pi}, \operatorname{c-Ind}_{\mathcal{K}_{d}}^{G}\left(\phi_{\Xi}\right)\right) \neq\{0\}
$$

- Up to isomorphism, c- $\operatorname{Ind}_{\mathcal{K}_{d}}^{G}\left(\phi_{\Xi}\right)$ contains $(q+1) q^{(d-1)}$ distinct classes of irreducible supercuspidal representations $\left(\sigma, V_{\sigma}\right)$. In particular, the number of distinct irreducible supercuspidal representations of depth $d$ which are induced from $\mathcal{K}$ is:

$$
\frac{(q-1)}{2}(q+1) q^{(d-1)}
$$

- The formal degree of any irreducible $\left(\sigma, V_{\sigma}\right)$ occurring in c-Ind $\mathcal{K}_{d}^{G}\left(\phi_{\Xi}\right)$ is:

$$
d_{\sigma}=\frac{1}{\operatorname{meas}(\mathcal{K})}(q-1) q q^{(d-1)}
$$

- The multiplicity in c- $\operatorname{Ind}_{\mathcal{K}_{d}}^{G}\left(\phi_{\Xi}\right)$ of any $\left(\sigma, V_{\sigma}\right)$ occurring in it is $q^{(d-1)}$; in particular is independent of $\sigma$.

(iv) For an elliptic character $\phi_{\Xi}$ of $\mathcal{K}_{d} / \mathcal{K}_{d+1}$, let $S_{\Xi}$ denote the set of $(q+1) q^{(d-1)}$ classes of irreducible supercuspidal representations occurring in $c-\operatorname{Ind}_{\mathcal{K}_{d}}^{G}\left(\phi_{\Xi}\right)$, and let $\Theta_{\Xi}$ denote the character of the representation c-Ind $\operatorname{\mathcal {I}}_{d}^{G}\left(\phi_{\Xi}\right)$. We have:

$$
\Theta_{\Xi}=q^{(d-1)} \sum_{\sigma \in S_{\Xi}} \Theta_{\sigma}
$$

By Harish-Chandra's character formula $[\mathrm{HC}]$ for induction from an open compact subgroup, $\Theta_{\Xi}$ is supported on $\operatorname{Ad}(G)\left(\mathcal{K}_{d}\right)$. If the cosets $\Xi_{1}$ and $\Xi_{2}$ belong to the same $\mathcal{K}$-orbit, then $\mathrm{c}-\operatorname{Ind}_{\mathcal{I}_{d}}^{G}\left(\phi_{\Xi_{1}}\right)$ and $\mathrm{c}$-Ind $\mathrm{In}_{\mathcal{I}_{d}}^{G}\left(\phi_{\Xi_{2}}\right)$ are equivalent representations, and therefore $\Theta_{\Xi_{1}}=\Theta_{\Xi_{2}}$.

Set

$\tau:=\sum_{\Xi} \phi_{\Xi}$ the sum of the elliptic characters of $\mathcal{K}_{d}$ (modulo $\mathcal{K}_{d+1}$ ),

and let $\Theta_{\tau}$ denote the character of the induced representation $c-\operatorname{Ind}_{\mathcal{K}_{d}}^{G}(\tau)$. We have:

$$
\Theta_{\tau}=\sum_{\Xi} \Theta_{\Xi}=\sum_{\Xi} q^{(d-1)} \sum_{\sigma \in S_{\Xi}} \Theta_{\sigma}=(q-1) q q^{(d-1)} \sum_{\rho(\sigma)=d} \Theta_{\sigma}
$$


and so,

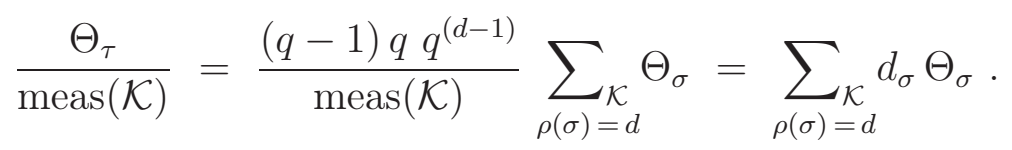

The sum $\sum_{\mathcal{K}}$ is over the classes of irreducible supercuspidal representations of depth $d$ which can be induced from $\mathcal{K}$.

For the compact group $\mathcal{K}^{\prime}$, with the obviously transposed construction, we have the similar identity:

$$
\frac{\Theta_{\tau^{\prime}}}{\operatorname{meas}\left(\mathcal{K}^{\prime}\right)}=\sum_{\rho(\sigma)=d} d_{\sigma} \Theta_{\sigma}
$$

Whence,

$$
e_{d}^{\text {cusp }}=\sum_{\substack{\mathcal{K}^{\prime} \\ \rho(\sigma)=d}} d_{\sigma} \Theta_{\sigma}+\sum_{\substack{\mathcal{K} \\ \rho(\sigma)=d}} d_{\sigma} \Theta_{\sigma}=\frac{1}{\operatorname{meas}(\mathcal{K})}\left(\Theta_{\tau}+\Theta_{\tau^{\prime}}\right)
$$

6.2. The projector $e_{d}^{\text {cusp }}$. Let $Z$ denote the subgroup of scalar matrices in $\operatorname{GL}(2, F)$, and set

$$
\begin{aligned}
K & :=\operatorname{GL}\left(2, \mathcal{R}_{F}\right) \\
\mathfrak{k}: & =\mathfrak{g r}\left(2, \mathcal{R}_{F}\right) \\
G_{u} & :=\left\{g \in \mathrm{GL}(2, F) \mid \operatorname{val}_{F}(\operatorname{det}(g)) \text { is even }\right\}=Z K \mathrm{SL}(2, F) .
\end{aligned}
$$

Let $K_{n}$ be the usual congruence subgroup of $K$, and for $n \in \mathbb{Z}$, set $\mathfrak{k}_{n}=\varpi^{n} \mathfrak{k}$. In particular, there are natural maps

$$
K_{d} / K_{d+1} \simeq \mathfrak{k}_{d} / \mathfrak{k}_{d+1} \text { and }\left(\mathfrak{k}_{d} / \mathfrak{k}_{d+1}\right)^{-}=\mathfrak{k}_{-d} / \mathfrak{k}_{-d+1} \simeq \mathfrak{g l}\left(2, \mathbb{F}_{q}\right)
$$

The inclusion $\mathfrak{s l}\left(2, \mathbb{F}_{q}\right) \subset \mathfrak{g l}\left(2, \mathbb{F}_{q}\right)$ allows us to canonically extend the previous subsection characters $\phi_{\Xi}$ of $\mathcal{K}_{d}$ (trivial $\mathcal{K}_{d+1}$ ), to characters of $Z K_{d}$ (trivial $Z \mathcal{K}_{d+1}$ ). We do this, and consequently get a canonical extension of the character $\tau$ to $Z K_{d}$. We shall use the same symbol $\tau$ to denote the extension. We recall:

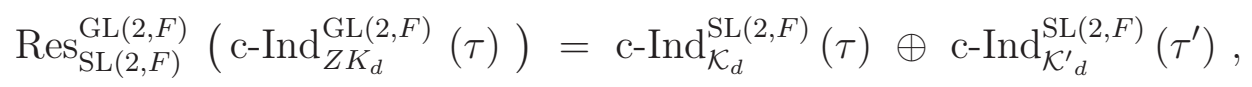

and therefore,

$$
e_{d}^{\text {cusp }}=\frac{1}{\operatorname{meas}(\mathcal{K})} \cdot \text { restriction to } \operatorname{SL}(2, F) \text { of the character of c-Ind } \operatorname{ILK}_{d}^{\mathrm{GL}(2, F)}(\tau)
$$




\subsection{The projector $e_{d}^{\text {cusp }}$ on split and ramified elliptic tori.}

If $y \in G$ is a regular element which is split or ramified elliptic, and $\pi$ is an irreducible supercuspidal representation of depth $d$, the Sally-Shalika [SS] character tables lists the character value $\Theta_{\pi}(y)$ as zero unless the eigenvalues $\alpha, \alpha^{-1}$ of $y$ have $\left|\alpha-\alpha^{-1}\right|<q^{-d}$. Furthermore, when $\left|\alpha-\alpha^{-1}\right|<q^{-d}$, the value $\Theta_{\pi}(y)$ depends only on $\left|\alpha-\alpha^{-1}\right|$ and $d$. By (6.1.1) and (6.1.2), it follows:

$$
\begin{aligned}
e_{d}^{\text {cusp }}(y) & =\# \text { irreducible supercuspidals } \cdot \text { formal degree } \cdot \Theta_{\pi}(y) \\
& =(q-1)(q+1) q^{(d-1)} \frac{1}{\operatorname{meas}(\mathcal{K})}(q-1) q q^{(d-1)} \Theta_{\pi}(y) .
\end{aligned}
$$

Case $y$ split: If $y$ is a (compact) split element with eigenvalues $\alpha, \alpha^{-1}$, then, by Table 2 (page 1235) of Sally-Shalika [SS], the character value of a depth $d$ irreducible supercuspidal (unramified) representation $\pi$ is:

$$
\Theta_{\pi}(y)= \begin{cases}0 & \text { when } \alpha \notin 1+\wp_{F}^{d+1} \\ \frac{1}{\left|\alpha-\alpha^{-1}\right|}-q^{d} & \text { for } \alpha \in\left(1+\wp_{F}^{d+1}\right)\end{cases}
$$

so, we get

$$
e_{d}^{\text {cusp }}(y)=(q-1)(q+1)\left\{\begin{array}{l}
0 \quad \text { when } \alpha \notin 1+\wp_{F}^{d+1}, \text { i.e., }|1-\alpha|>\frac{1}{q^{d+1}} \\
(q-1) q q^{2(d-1)}\left(\frac{1}{\left|\alpha-\alpha^{-1}\right|}-q^{d}\right) \text { for }|1-\alpha| \leq \frac{1}{q^{d+1}}
\end{array}\right.
$$

Case $y$ ramified elliptic: If $y$ is a ramified elliptic element with eigenvalues $\alpha, \alpha^{-1}$, let $E$ be the quadratic extension of $F$ containing $\alpha$. Then, the character value of a depth $d$ cuspidal (unramified) representation $\pi$ is

$$
\Theta_{\pi}(y)= \begin{cases}0 & \text { when } \alpha \notin\left(1+\wp_{E}^{2 d+1}\right) \\ -q^{d} & \text { for } \alpha \in\left(1+\wp_{E}^{2 d+1}\right), \text { i.e., }|1-\alpha| \leq \frac{1}{q^{d+\frac{1}{2}}}\end{cases}
$$

so, we get

$$
e_{d}^{\text {cusp }}(y)=(q-1)(q+1) \begin{cases}0 & \text { when } \alpha \notin 1+\wp_{E}^{2 d+1}, \\ (q-1) q q^{2(d-1)} q^{d}(-1) & \text { for }|1-\alpha|_{E} \leq \frac{1}{q^{d+\frac{1}{2}}} .\end{cases}
$$


6.4. The projector $e_{d}^{\text {cusp }}$ on unramified elliptic tori. Suppose $y \in G$ is a regular element whose eigenvalues $\alpha, \alpha^{-1}$ bekong to the unramified quadratic extension $E / F$. We deduce from the formula (6.2.3) that

$$
e_{d}^{\text {cusp }}(y)=0 \quad \text { unless } \alpha \in 1+\wp_{E}^{d} \text {. }
$$

Subcase $\left|\alpha-\alpha^{-1}\right|=q^{-d}$ : The element $y$ has a conjugate in the set $K_{d} \backslash K_{d+1}$. We may and do assume $y$ is in $K_{d} \backslash K_{d+1}$.

Lemma 6.4.1. Let $\psi$ be a non-trivial character of $\mathbb{F}_{q}$. Suppose $z \in \mathfrak{s l}\left(2, \mathbb{F}_{q}\right)$ is a elliptic element. Then,

$$
\sum_{\substack{e \in \mathfrak{s r}\left(2, \mathbb{F}_{q}\right) \\ e \text { elliptic }}} \psi(\operatorname{trace}(z e))=q .
$$

Proof. The orbit of an elliptic element has a representive of the form $\left[\begin{array}{ll}0 & 1 \\ u & 0\end{array}\right]$, with $u$ a non-square. A parametrization of the elliptic elements is:

$$
\operatorname{Ad}\left(\left[\begin{array}{ll}
a & b \\
0 & 1
\end{array}\right]\right)\left[\begin{array}{ll}
0 & 1 \\
u & 0
\end{array}\right] \text {, with } u \text { a non-square, } a \text { non-zero, and } b \text { arbitrary. }
$$

We take $z=\left[\begin{array}{ll}0 & 1 \\ \epsilon & 0\end{array}\right]$, with $\epsilon$ a non-square. Then, the sum (6.4.2) becomes

$$
\begin{aligned}
& \sum_{\substack{u \text { non-square } \\
a \neq 0, b}} \psi\left(u a^{-1}+\epsilon a-\epsilon b^{2} u a^{-1}\right)=\sum_{a} \psi(\epsilon a) \sum_{u} \psi\left(u a^{-1}\right) \sum_{b} \psi\left(-\epsilon b^{2} u a^{-1}\right) \\
& \quad=\sum_{a} \psi(\epsilon a) \sum_{v \text { non-square }} \psi(v a) \sum_{c} \psi\left(-a c^{2}\right) .
\end{aligned}
$$

Let $\psi_{a}$ be the character $\psi_{a}(x)=\psi(a x)$, and let sgn denote the quadratic character on $\mathbb{F}_{q}$. Then, $\sum_{c} \psi\left(-a c^{2} a\right)=G\left(\psi_{-a}, \operatorname{sgn}\right)=\operatorname{sgn}(-a) G(\psi, \operatorname{sgn})$, a Gauss sum. So, the sum (6.4.2) equals 


$$
\begin{aligned}
\sum_{a \neq 0} \psi(\epsilon a) & \left(\sum_{v \text { non-square }} \psi(v a)\right) \operatorname{sgn}(-a) G(\psi, \operatorname{sgn}) \\
& =\sum_{a \neq 0} \psi(\epsilon a) \frac{1}{2}\left(-1-G\left(\psi_{a}, \operatorname{sgn}\right)\right) \operatorname{sgn}(-a) G(\psi, \operatorname{sgn}) \\
& =-\frac{1}{2} \sum_{a \neq 0}(\psi(\epsilon a) \operatorname{sgn}(-a) G(\psi, \operatorname{sgn})+\psi(\epsilon a) q) \\
& =-\frac{1}{2}\left(G(\psi, \operatorname{sgn}) \operatorname{sgn}(-1) \operatorname{sgn}(\epsilon) \sum_{a \neq 0} \psi(a) \operatorname{sgn}(a)+\sum_{a \neq 0} \psi(\epsilon a) q\right) \\
& =-\frac{1}{2}(-q+(-q))=q
\end{aligned}
$$

Here, we have used elementary properties of Gauss sums [IR].

Corollary 6.4.3. Let $\tau$ be the sum (6.1.3) of the elliptic characters of $\mathcal{K}_{d}$ (modulo $\mathcal{K}_{d+1}$ ). If $y \in \mathcal{K}_{d} \backslash \mathcal{K}_{d+1}$ is an elliptic element whose eigenvalues $\alpha, \alpha^{-1}$ satisfy $\left|\alpha-\alpha^{-1}\right|=q^{-d}$, then

$$
\tau(y)=q .
$$

As already mentioned above (6.2.3), $\tau$ has a canonical extension to $Z K_{d}$. Let $\dot{\tau}$ denote the extension of the function $\tau$ to $\mathrm{GL}(2, F)$ which is zero outside $Z K_{d}$. Suppose $y \in \mathcal{K}_{d} \backslash \mathcal{K}_{d+1}$ is unramified elliptic. Harish-Chandra's formula [HC] for the right side of (6.2.3)

$$
\begin{aligned}
& e_{d}^{\text {cusp }}(y)=\sum_{g Z K_{d} \in \operatorname{GL}(2, F) / Z K_{d}} \dot{\tau}\left(g y g^{-1}\right) \quad \text { for } y \in \mathcal{K}_{d} \backslash \mathcal{K}_{d+1} \text { elliptic } \\
& \quad=\sum_{g Z K_{d} \in Z K / Z K_{d}} \dot{\tau}\left(g y g^{-1}\right)=\sum_{g Z K_{d} \in Z K / Z K_{d}} \dot{\tau}(y)=\left[Z K: Z K_{d}\right] \tau(y) \\
& =(q+1)(q-1) q\left(q^{3}\right)^{(d-1)} \tau(y)=(q+1)(q-1) q^{2} q^{3(d-1)}
\end{aligned}
$$

Subcase $\left|\alpha-\alpha^{-1}\right|<q^{-d}$ : Suppose $\pi$ is an irreducible supercupsidal representation compact induced from the representation $\kappa$ of $\mathcal{K}$. Let $\kappa^{\prime}$ be the $\mathrm{GL}(2, F)$ conjugation of $\kappa$ to

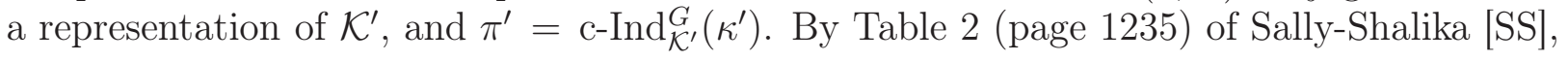

and so

$$
\Theta_{\pi}(y)+\Theta_{\pi^{\prime}}(y)=-2 q^{d}
$$

$$
\begin{aligned}
e_{d}^{\text {cusp }}(y) & =\frac{(q-1)}{2}(q+1) q^{(d-1)} \cdot(q-1) q q^{(d-1)} \cdot(-2) q^{d} \\
& =(q-1)(q+1)(q-1) q^{3 d-1}(-1) \quad \text { when }\left|\alpha-\alpha^{-1}\right|<q^{-d}
\end{aligned}
$$




\begin{tabular}{|c|c|}
\hline & Table $2 d \geq 0$ integral \\
\hline \multicolumn{2}{|r|}{$y$ has eigenvalues $\alpha, \alpha^{-1}: \quad$ value of $\frac{1}{(q-1)(q+1)} e_{d}^{\text {cusp }}(y)$} \\
\hline$y$ split & $\left\{\begin{array}{l}0 \quad \text { when } \alpha \notin 1+\wp_{F}^{d+1} \text {, i.e., }|1-\alpha|>\frac{1}{q^{d+1}} \\
(q-1) q q^{2(d-1)}\left(\frac{1}{\left|\alpha-\alpha^{-1}\right|}-q^{d}\right) \quad \text { for }|1-\alpha| \leq \frac{1}{q^{d+1}}\end{array}\right.$ \\
\hline $\begin{array}{l}y \text { ramified } \\
\text { elliptic }\end{array}$ & $\left\{\begin{array}{l}0 \quad \text { when } \alpha \notin 1+\wp_{E}^{2 d+1} \text {, i.e., }|1-\alpha|>\frac{1}{q^{d+\frac{1}{2}}} \\
(q-1) q q^{2(d-1)} q^{d}(-1) \quad \text { for }|1-\alpha|_{E} \leq \frac{1}{q^{d+\frac{1}{2}}}\end{array}\right.$ \\
\hline $\begin{array}{l}y \text { unramified } \\
\text { elliptic }\end{array}$ & $\left\{\begin{array}{l}0 \quad \text { when } \alpha \notin 1+\wp_{E}^{d+1} \text {, i.e., }|1-\alpha|>\frac{1}{q^{d}} \\
q^{(3 d-1)} \quad \text { when }|1-\alpha|=\frac{1}{q^{d}} \\
(q-1) q^{(3 d-1)}(-1) \quad \text { for }|1-\alpha|<\frac{1}{q^{d}}\end{array}\right.$ \\
\hline
\end{tabular}

\section{Half-Integral DePth Supercuspidal REPRESEntations}

We abbreviate the Iwahori subgroup $G_{x_{01}}$ and its filtration subgroups $G_{x_{01}, r}$ as $\mathcal{I}$ and $\mathcal{I}_{r}$ respectively.

Suppose $d \in \frac{1}{2}+\mathbb{N}$ is a positive half-integer. A Bernstein component $\Omega$ of depth $d$ is the equivalence class of an irreducible supercuspidal representation $\pi$. Set

$$
d^{+}:=d+\frac{1}{2} .
$$

We recall:

(i) The group $\mathcal{I}_{d} / \mathcal{I}_{d^{+}}$has $(q-1)^{2}$ non-degenerate characters. Under the adjoint action of $\mathcal{I}$, these non-degenerate characters are partitioned into $2(q-1)$ orbits with $\frac{(q-1)}{2}$ characters in an orbit. 
(ii) If $\left(\pi, V_{\pi}\right)$ is an irreducible supercuspidal representation, and its depth $\rho(\pi)$ equals $d$, then the subspace of $\mathcal{I}_{d^{+}}$-fixed vectors, $V_{\pi}^{\mathcal{I}_{d^{+}}}$, is non-zero, and is, since $\mathcal{I}$ normalizes the subgroup $\mathcal{I}_{d^{+}}, \mathcal{I}$-invariant. The characters $\phi_{X}$ of $\mathcal{I}_{d}$ (modulo $\mathcal{I}_{d^{+}}$) which appear in $V_{\pi}^{\mathcal{I}_{d^{+}}}$are non-degenerate. By Clifford theory, the set

$$
\left\{\phi_{\Xi} \mid \phi_{\Xi} \text { appears in } V_{\pi}^{\mathcal{I}_{d^{+}}}\right\}
$$

is a single $\mathcal{I}$-orbit.

The formal degree of $\pi$ is:

$$
d_{\pi}=\frac{(q+1)}{\operatorname{meas}(\mathcal{K})} \frac{(q-1)}{2} q^{d-\frac{1}{2}}
$$

(iii) For any non-degenerate character $\phi_{\Xi}$ of $\mathcal{I}_{d} / \mathcal{I}_{d^{+}}$, the compactly supported induced representation

$$
\mathrm{c}-\operatorname{Ind}_{\mathcal{I}_{d}}^{G}\left(\phi_{\Xi}\right)
$$

is a finite length (completely reducible) supercuspidal representation. If $\left(\pi, V_{\pi}\right)$ is an irreducible supercuspidal representation as in part (i), i.e., $V_{\pi}^{\mathcal{I}_{d^{+}}}$contains the (nondegenerate) character $\phi_{\Xi}$, then by Frobenius reciprocity:

$$
\operatorname{Hom}_{G}\left(V_{\pi}, \mathrm{c}-\operatorname{Ind}_{\mathcal{I}_{d}}^{G}\left(\phi_{\Xi}\right)\right) \neq\{0\}
$$

Furthermore:

- Up to isomorphism, c- $\operatorname{Ind}_{\mathcal{I}_{d}}^{G}\left(\phi_{\Xi}\right)$ contains $2 q^{\left(d-\frac{1}{2}\right)}$ distinct classes of irreducible supercuspidal representations $\left(\sigma, V_{\sigma}\right)$.

- The multiplicity in c- $\operatorname{Ind}_{\mathcal{I}_{d}}^{G}\left(\phi_{\Xi}\right)$ of any $\left(\sigma, V_{\sigma}\right)$ occurring in it is $q^{\left(d-\frac{1}{2}\right)}$; in particular, the multiplicity is independent of $\sigma$

(iv) For a non-degenerate character $\phi_{\Xi}$, let $S_{\Xi}$ denote the set of these $2 q^{\left(d-\frac{1}{2}\right)}$ classes of irreducible supercuspidal representations, and let $\Theta_{\Xi}$ denote the character of the representation $\mathrm{c}-\operatorname{Ind}_{\mathcal{I}_{d}}^{G}\left(\phi_{\Xi}\right)$. We have:

$$
\Theta_{\Xi}=q^{\left(d-\frac{1}{2}\right)} \sum_{\sigma \in S_{\Xi}} \Theta_{\sigma} .
$$

By Harish-Chandra's character formula $[\mathrm{HC}]$ for induction from an open compact subgroup, $\Theta_{\Xi}$ is supported on $\operatorname{Ad}(G)\left(\mathcal{I}_{d}\right)$. If the cosets $\Xi$ and $\Xi^{\prime}$ belong to the same $\mathcal{I}$-orbit, then $\mathrm{c}-\operatorname{Ind}_{\mathcal{I}_{d}}^{G}\left(\phi_{\Xi}\right)$ and $\mathrm{c}-\operatorname{Ind}_{\mathcal{I}_{d}}^{G}\left(\phi_{\Xi^{\prime}}\right)$ are equivalent representations, and so $\Theta_{\Xi}=\Theta_{\Xi^{\prime}}$. Set

$\tau:=\sum_{\Xi} \phi_{\Xi}$ the sum of the non-degenerate characters of $\mathcal{I}_{d}$ (modulo $\mathcal{I}_{d^{+}}$),

and let $\Theta_{\tau}$ denote the character of the (compactly supported) induced representation c- $\operatorname{Ind}_{\mathcal{I}_{d}}^{G}(\tau)$. We have: 


$$
\begin{aligned}
\Theta_{\tau} & =\sum_{\Xi} \Theta_{\Xi}=\sum_{\Xi} q^{\left(d-\frac{1}{2}\right)} \sum_{\sigma \in S_{\Xi}} \Theta_{\sigma} \\
& =\frac{(q-1)}{2} q^{\left(d-\frac{1}{2}\right)} \sum_{\rho(\sigma)=d} \Theta_{\sigma} .
\end{aligned}
$$

Whence,

$$
\Theta_{\tau}=\frac{\operatorname{meas}(\mathcal{K})}{(q+1)}\left(\frac{(q+1)}{\operatorname{meas}(\mathcal{K})} \frac{(q-1)}{2} q^{\left(d-\frac{1}{2}\right)}\right) \sum_{\rho(\sigma)=d} \Theta_{\sigma}
$$

i.e.,

$$
e_{d}=\frac{(q+1)}{\operatorname{meas}(\mathcal{K})} \Theta_{\tau}
$$

As already mentioned above for $\Theta_{\Xi}$, by Harish-Chandra's formula [HC] for supercuspidal representations obtained via compact induction, we have:

$$
\operatorname{support}\left(e_{d}\right) \subset \operatorname{Ad}(G)\left(\mathcal{I}_{d}\right) .
$$

This support condition allows us to computer $e_{d}$ rather efficiently. Note for $d>0$, and residual characteristic $p$ odd, the set $\mathcal{I}_{d}$ is contained in $\mathcal{U}^{\text {top }}$. Whence the support of $e_{d}$ is within the set of topologically unipotent elements.

Suppose $y \in G$ is regular semisimple element. Let $\alpha, \alpha^{-1}$ be the roots of the characteristic polynomial of $y$. By the support condition:

$$
e_{d}(y)=0 \quad \text { when } \quad\left|\alpha-\alpha^{-1}\right|>q^{-d} .
$$

When $\left|\alpha-\alpha^{-1}\right| \leq q^{-d}$, we consider three cases for $y$ : split, elliptic unramified and elliptic ramified.

Case $y$ split or elliptic unramified: Here, the eigenvalues of $y$ belong to either $F$ or an unramified quadratic extension, and therefore $\left|\alpha-\alpha^{-1}\right|$ is a (positive) integral power of $\frac{1}{q}$, so $\left|\alpha-\alpha^{-1}\right| \leq q^{-d}$ in fact means $\left|\alpha-\alpha^{-1}\right|<q^{-d}$. By the Sally-Shalika character tables [SS], if $\pi$ is an irreducible supercuspidal representation of depth $d$ :

$\Theta_{\pi}(y)= \begin{cases}0 & y \text { split or unramified elliptic, and }\left|\alpha-\alpha^{-1}\right|>q^{-d} \\ \frac{1}{\left|\alpha-\alpha^{-1}\right|}-\frac{1}{2} q^{d+\frac{1}{2}\left(\frac{q+1}{q}\right)} & \text { when } y \text { is split and }\left|\alpha-\alpha^{-1}\right| \leq q^{-d} \\ -\frac{1}{2} q^{d+\frac{1}{2}\left(\frac{q+1}{q}\right)} & y \text { unramified elliptic, and }\left|\alpha-\alpha^{-1}\right| \leq q^{-d}\end{cases}$

So, 
$\frac{\operatorname{meas}(\mathcal{K})}{(q-1)(q+1)} e_{d}(y)=\left\{\begin{array}{r}\begin{array}{r}0 \\ \frac{\left(2(q-1) q^{2\left(d-\frac{1}{2}\right)}\right)}{\left|\alpha-\alpha^{-1}\right|}-(q-1)(q+1) q^{3\left(d-\frac{1}{2}\right)} \\ \text { when } y \text { is split and }\left|\alpha-\alpha^{-1}\right| \leq q^{-d} \\ (q-1)(q+1) q^{3\left(d-\frac{1}{2}\right)}(-1) \\ y \text { unramified elliptic and }\left|\alpha-\alpha^{-1}\right| \leq q^{-d}\end{array}\end{array}\right.$

Case $y$ ramified: As already mentioned, by the support condition, we may and do assume $\left|\alpha-\alpha^{-1}\right| \leq q^{-d}$. We consider two subcases depending on whether:

$$
\left|\alpha-\alpha^{-1}\right|<q^{-d} \quad \text { or } \quad\left|\alpha-\alpha^{-1}\right|=q^{-d} \text {. }
$$

Subcase $\left|\alpha-\alpha^{-1}\right|=q^{-d}$ : The element $y$ has a conjugate in the set $\mathcal{I}_{d} \backslash \mathcal{I}_{d+\frac{1}{2}}$. We may and do assume $y$ is in $\mathcal{I}_{d} \backslash \mathcal{I}_{d+\frac{1}{2}}$. We remark that under the isomorphism of $\mathcal{I}_{d} / \mathcal{I}_{d+\frac{1}{2}}$ with $\mathfrak{g}_{x_{01, d}} / \mathfrak{g}_{x_{01,}{ }_{d+\frac{1}{2}}}$, the coset $y$ is a non-degenerate coset.

Lemma 7.4. Let $\psi$ be a non-trivial character of $\mathbb{F}_{q}$. Suppose $u, v \in\left(\mathbb{F}_{q}\right)^{\times}$. Then,

$$
\sum_{a, b \in\left(\mathbb{F}_{q}\right)^{\times}} \psi(u a) \psi(v b)=1
$$

Proof. By changes of variables

$$
\sum_{a, b \in\left(\mathbb{F}_{q}\right)^{\times}} \psi(u a) \psi(v b)=\left(\sum_{c \in\left(\mathbb{F}_{q}\right)^{\times}} \psi(c)\right)^{2}=(-1)^{2}=1 .
$$

Corollary 7.5. If $y \in \mathcal{I}_{d} \backslash \mathcal{I}_{d+\frac{1}{2}}$ is a ramified elliptic element whose eigenvalues $\alpha, \alpha^{-1}$ satisfy $\left|\alpha-\alpha^{-1}\right|=q^{-d}$, then

$$
\tau(y)=1 .
$$

Let $\dot{\tau}$ denote the extension of $\tau$ to a function on $G$ which is zero outside $\mathcal{I}_{d}$. HarishChandra's formula $[\mathrm{HC}]$ for the character $\Theta_{\tau}$ is:

$$
\Theta_{\tau}(x)=\sum_{g \mathcal{I}_{d} \in G / \mathcal{I}_{d}} \dot{\tau}\left(g x g^{-1}\right) .
$$

Since $y \in \mathcal{I}_{d}$ has the property that under the isomorphism $\mathcal{I}_{d} / \mathcal{I}_{d+\frac{1}{2}} \simeq \mathfrak{g}_{x_{01, d}} / \mathfrak{g}_{x_{01}, d+\frac{1}{2}}$ it corresponds to a non-degenerate coset, the only $g \mathcal{I}_{d}$ satisfying $g$ y $g^{-1} \in \mathcal{I}_{d}$ is when $g \in \mathcal{I}$. 
Whence,

$$
\begin{aligned}
\Theta_{\tau}(y) & =\sum_{g \mathcal{I}_{d} \in G / \mathcal{I}_{d}} \dot{\tau}\left(g y g^{-1}\right)=\sum_{g \mathcal{I}_{d} \in \mathcal{I} / \mathcal{I}_{d}} \dot{\tau}\left(g y g^{-1}\right) \\
& =\left[\mathcal{I}: \mathcal{I}_{d}\right] \dot{\tau}(y)=(q-1) q^{3\left(d-\frac{1}{2}\right)} \tau(y)=(q-1) q^{3\left(d-\frac{1}{2}\right)}
\end{aligned}
$$

and

$$
\frac{\operatorname{meas}(\mathcal{K})}{(q-1)(q+1)} e_{d}(y)=q^{3\left(d-\frac{1}{2}\right)} \quad \text { when } \quad\left|\alpha-\alpha^{-1}\right|=q^{-d}
$$

Subcase $\left|\alpha-\alpha^{-1}\right|<q^{-d}$ : We note irreducible supercuspidal representations of depth $d$ come in pairs $\pi$ and $\pi^{\prime}$, i.e., an $L$-packet. In the Sally-Shalika [SS] parameterization of ramified irreducible supercuspidal representation, each element of the pair corresponds to taking one of two classes of additive characters of $F$, and their character table gives:

$$
\left(\Theta_{\pi}+\Theta_{\pi^{\prime}}\right)(y)=-q^{d+\frac{1}{2}} \frac{(q+1)}{q}=-q^{d-\frac{1}{2}}(q+1) \quad \text { when } \quad|1-\alpha|<q^{-d}
$$
so,

$$
\left(e_{\pi}+e_{\pi^{\prime}}\right)(y)=\frac{(q-1)(q+1) q^{\left(d-\frac{1}{2}\right)}}{2 \operatorname{meas}(\mathcal{K})} \cdot\left(-q^{d-\frac{1}{2}}(q+1)\right)
$$

Whence,

$$
\begin{aligned}
e_{d}(y) & =2(q-1) q^{\left(d-\frac{1}{2}\right)}\left(\frac{(q-1)(q+1) q^{\left(d-\frac{1}{2}\right)}}{2 \operatorname{meas}(\mathcal{K})}\right) \cdot\left(-q^{d-\frac{1}{2}}(q+1)\right) \\
& =-\frac{(q-1)^{2}(q+1)^{2}}{\operatorname{meas}(\mathcal{K})} q^{3\left(d-\frac{1}{2}\right)}
\end{aligned}
$$

i.e.,

$$
\frac{\operatorname{meas}(\mathcal{K})}{(q-1)(q+1)} e_{d}(y)=(q-1)(q+1) q^{3\left(d-\frac{1}{2}\right)}(-1)
$$




\begin{tabular}{|c|c|}
\hline & Table $3 \quad d \in \mathbb{N}$ half-integral \\
\hline & $y$ has eigenvalues $\alpha, \alpha^{-1}: \quad$ value of $\frac{1}{(q-1)(q+1)} e_{d}(y)$ \\
\hline$y$ split & $\left\{\begin{array}{l}0 \quad \text { when } \alpha \notin 1+\wp_{F}^{d+\frac{1}{2}} \text {, i.e., }|1-\alpha|>\frac{1}{q^{d}} \\
\frac{\left(2(q-1) q^{2\left(d-\frac{1}{2}\right)}\right)}{\left|\alpha-\alpha^{-1}\right|}-(q-1)(q+1) q^{3\left(d-\frac{1}{2}\right)} \quad \text { when }\left|\alpha-\alpha^{-1}\right| \leq q^{-d}\end{array}\right.$ \\
\hline $\begin{array}{l}y \text { ramified } \\
\text { elliptic }\end{array}$ & $\begin{cases}0 & \text { when }|1-\alpha|>\frac{1}{q^{d}} \\
q^{3\left(d-\frac{1}{2}\right)} & \text { for }|1-\alpha|_{E}=\frac{1}{q^{d}} \\
(q-1)(q+1) q^{3\left(d-\frac{1}{2}\right)}(-1) \quad \text { for }|1-\alpha|_{E}<\frac{1}{q^{d}}\end{cases}$ \\
\hline $\begin{array}{l}y \text { unramified } \\
\text { elliptic }\end{array}$ & $\left\{\begin{array}{l}0 \quad \text { when }|1-\alpha|>\frac{1}{q^{d}} \\
(q-1)(q+1) q^{3\left(d-\frac{1}{2}\right)}(-1) \quad \text { for }|1-\alpha|<\frac{1}{q^{d}}\end{array}\right.$ \\
\hline
\end{tabular}

\section{The Main Result}

For convenience in numbering, for $k \in \frac{1}{2} \mathbb{N}$, set

$$
\sigma_{k}:=e_{0}+e_{\frac{1}{2}}+\cdots+e_{k}
$$

We note in particular $\sigma_{0}=e_{0}$.

Theorem 8.2. For $k \in \frac{1}{2} \mathbb{N}$, set $k^{+}:=k+\frac{1}{2}$. Under the assumption the p-adic field $F$ has odd residue characteristic, we have $\operatorname{supp}\left(\sigma_{k}\right) \subset \mathcal{U}_{k^{+}}^{\text {top }} . O n \mathcal{U}_{k^{+}}^{\text {top }}$ :

- When $k$ is integral: 


$$
\sigma_{k}(y)=\left(q^{2}-1\right) q^{3 k}\left\{\begin{array}{l}
\left(\frac{2 q^{-k}}{\left|\alpha-\alpha^{-1}\right|_{F}}-1\right) \\
\text { when } y \text { is split with eigenvalues } \alpha, \alpha^{-1} \\
-1 \quad \text { when } y \text { is elliptic }
\end{array}\right.
$$

- When $k$ is half-integral:

$$
\sigma_{k}(y)=\left(q^{2}-1\right) q^{3 k+\frac{1}{2}}\left\{\begin{array}{c}
\left(\frac{2 q^{-\left(k+\frac{1}{2}\right)}}{\left|\alpha-\alpha^{-1}\right|_{F}}-1\right) \\
\text { when } y \text { is split with eigenvalues } \alpha, \alpha^{-1} \\
-1 \quad \text { when } y \text { is elliptic }
\end{array}\right.
$$

Proof. The proof is induction on the depth: The values of $\sigma_{0}=e_{0}$ are given in Table 1 . Given $\sigma_{k}$, we compute $\sigma_{k^{+}}=\sigma_{k}+e_{k^{+}}$via Table 3 when $k$ is integral, and via (4.3) and Table 2 when $k$ is half-integral.

For $k \in \frac{1}{2} \mathbb{N}$, we note the series defining the exponential and logarithm maps between $\mathfrak{g}$ and $G$ converge for $k$ sufficiently large. We take $k_{0} \in \frac{1}{2} \mathbb{N}$ so that:

$$
\text { exp and } \log \text { are bijections between } \mathfrak{g}_{k} \text { and } G_{k} \text { when } k \geq k_{o} \text {. }
$$

Corollary 8.4. Under condition (8.3), so that $\sigma_{k} \circ \exp$ is defined and has support in $\mathcal{N}_{k^{+}}^{\text {top }}$, if $Y \in \mathcal{N}_{k^{+}}^{\text {top }}$ has eigenvalues $\pm \lambda$, then :

- When $k$ is integral and $Y \in \mathfrak{g}_{k^{+}}$:

$$
\sigma_{k} \circ \exp (Y)=\left(q^{2}-1\right) q^{3 k}\left\{\begin{array}{r}
\left(\frac{2 q^{-k}}{|\lambda|_{F}}-1\right) \\
\text { when } y \text { is split } \\
-1 \quad \\
\text { when } y \text { is elliptic }
\end{array}\right.
$$

- When $k$ is half-integral and $Y \in \mathfrak{g}_{k^{+}}$:

$$
\sigma_{k} \circ \exp (Y)=\left(q^{2}-1\right) q^{3 k+\frac{1}{2}}\left\{\begin{array}{r}
\left(\frac{2 q^{-\left(k+\frac{1}{2}\right)}}{|\lambda|_{F}}-1\right) \\
\text { when } y \text { is split } \\
-1 \quad \\
\text { when } y \text { is elliptic }
\end{array}\right.
$$


In particular, $\sigma_{k+1} \circ \exp$ and $\sigma_{k} \circ \exp$ satisfy the homogeneity relation:

$$
\left(\sigma_{k+1} \circ \exp \right)(\varpi Y)=q^{3}\left(\sigma_{k} \circ \exp \right)(Y)
$$

Remarks: (i) Examination of the explicit formulae for $\sigma_{k}$ and $\sigma_{k} \circ$ exp, shows these distributions depend only on the characteristic polynomial of the input, and therefore are stable distributions.

(ii) The formula for $\sigma_{0}=\epsilon_{0}$ leads to the observation that it is the restriction of the Steinberg character to $\mathcal{U}^{\text {top }}$.

(iii) The transfer of $\sigma_{k}$ to $\sigma_{k}$ oexp is only valid when $k \geq k_{0}$, but the homogeneity relation (8.5) allows us to formally continue $\sigma_{k}$ o exp to the range $0 \leq k<k_{0}$, so the continuation for parameter has support in $\mathfrak{g}_{k^{+}}$, e.g., $\mathfrak{g}_{0^{+}}=\mathcal{N}^{\text {top }}$ when $k=0$. This is analogous to the behavior of $\sigma_{k}$ 's in the same range.

(iv) The power 3 of the factor $q^{3}$ should be viewed as the dimension of $\mathfrak{g}$. Under Fourier transform on the Lie algebra, the homogeneity relation (8.5) becomes a

$$
\mathrm{FT}\left(\sigma_{k+1} \circ \exp \right)\left(\varpi^{-1} Y\right)=\mathrm{FT}\left(\sigma_{k} \circ \exp \right)(Y) .
$$

To identify $\mathrm{FT}\left(\sigma_{k} \circ \exp \right)$, we note the following Proposition, whose proof is in the appendix:

Proposition. A.1 For $\mathfrak{g}=\mathfrak{s l}(2, F)$, we have

- The Fourier transforms $F T\left(1_{\mathfrak{g}_{0}}\right)$ and $F T\left(1_{\mathfrak{g}_{-\frac{1}{2}}}\right)$ have support in the sets $\mathfrak{g}_{0^{+}}:=\mathfrak{g}_{\frac{1}{2}}$ and $\mathfrak{g}_{\left(\frac{1}{2}\right)^{+}}:=\mathfrak{g}_{1}$ respectively. In particular, the support is contained in $\mathcal{N}^{\text {top }}$.

- For $k \geq 1$, the Fourier transform $F T\left(1_{\mathfrak{g}_{-k}}\right)$ has support in $\mathfrak{g}_{k^{+}}:=\mathfrak{g}_{k+\frac{1}{2}}$.

For a general connected reductive p-adic group, under conditions in which the exponential map takes $\mathcal{N}_{r}^{\text {top }}$ to $\mathcal{U}_{r}^{\text {top }}(r>0)$, Kim $[\mathrm{Ka}, \mathrm{Kb}]$, showed, for $X$ in $\mathfrak{g}_{\left(\frac{d}{2}\right)^{+}}$:

$$
\int_{\widehat{G}_{\leq d}^{\mathrm{temp}}} \Theta_{\pi}(\exp (X)) d \mu_{\mathrm{PM}}(\pi)=F T\left(1_{\mathfrak{g}_{-d}}\right)(X),
$$

where $\widehat{G}_{\leq d}^{\text {temp }}$ is the (classes of) irreducible tempered representations of depth less than or equal to $\bar{d}$. In this situation, for $\mathrm{SL}(2)$, we have

$$
\left.\sigma_{d} \circ \exp =F T\left(1_{\mathfrak{g}_{-d}}\right) \quad \text { (both sides have support in } \mathfrak{g}_{d^{+}}\right) .
$$

We conjecture, for $\mathrm{SL}(2)$, and more generally for any connected reductive p-adic group, this identity is true when the depth is sufficiently large. 


\section{APPENDix A.}

Here $G=\operatorname{SL}(2, F)$, and $\mathfrak{g}=\mathfrak{s l}(2, F)$. Suppose $\psi$ is an additive character of $F$ with conductor $\wp$. Let FT denote the Fourier transform on $\mathfrak{g}$, i.e., if $f \in C_{c}^{\infty}(\mathfrak{g})$ :

$$
\mathrm{FT}(f)(Y)=\int_{\mathfrak{g}} \psi(\operatorname{trace}(X Y)) f(X) d X .
$$

In this appendix we prove:

Proposition A.1. For $\mathfrak{g}=\mathfrak{s l}(2, F)$, we have

- The Fourier transforms $F T\left(1_{\mathfrak{g}_{0}}\right)$ and $F T\left(1_{\mathfrak{g}_{-\frac{1}{2}}}\right)$ have support in the sets $\mathfrak{g}_{0^{+}}:=\mathfrak{g}_{\frac{1}{2}}$ and $\mathfrak{g}_{\left(\frac{1}{2}\right)^{+}}:=\mathfrak{g}_{1}$ respectively. In particular, the support is contained in $\mathcal{N}^{\text {top }}$.

- For $k \geq 1$, the Fourier transform $F T\left(1_{\mathfrak{g}_{-k}}\right)$ has support in $\mathfrak{g}_{k^{+}}:=\mathfrak{g}_{k+\frac{1}{2}}$.

Proof. Since $1_{\mathfrak{g}_{0}}$, and $1_{\mathfrak{g}_{-\frac{1}{2}}}$ are $\operatorname{Ad}(G)$-invariant sets, their Fourier transforms are $\operatorname{Ad}(G)$ invariant. Therefore, it is sufficient to show the stated vanishing on any convenient element in an Adjoint orbit.

We prove the result for $1_{\mathfrak{g}_{0}}$ and remark our argument proof is easily adapted to also treat the case $1_{\mathfrak{g}_{-\frac{1}{2}}}$. We have

$$
\mathrm{FT}\left(1_{\mathfrak{g}_{0}}\right)(Y)=\mathrm{PV} \int_{\mathfrak{g}} \psi\left(\operatorname{trace}(X Y) 1_{\mathfrak{g}_{0}}(X) d X \quad\right. \text { (principal value). }
$$

We note that $\mathfrak{g}_{0}=\left\{X \in \mathfrak{g} \mid \operatorname{det}(X) \in \mathcal{R}_{F}\right\}$. Let $X=\left[\begin{array}{cc}a & b \\ c & -a\end{array}\right]$, and for integral $\ell$, set

$$
\mathcal{T}_{\ell}=\left\{X \in \mathfrak{g}_{0} \mid a, b, c \in \wp^{-\ell}\right\}=\left\{X \in \mathfrak{g} \mid \operatorname{det}(X) \in \mathcal{R}_{F} \text { and } a, b, c \in \wp^{\ell}\right\} .
$$

We show for $Y \notin \mathfrak{g}_{\frac{1}{2}}$ the integral

$$
\int_{\mathcal{T}_{\ell}} \psi(\operatorname{trace}(X Y)) 1_{\mathfrak{g} 0}(X) d X=\int_{\mathcal{T}_{\ell}} \psi(\operatorname{trace}(X Y)) d X \quad \text { vanishes for } \ell \text { large. }
$$

The Fourier transforms $\mathrm{FT}\left(1_{\mathfrak{g}_{j}}\right)$ are invariant under the Adjoint action of $\mathrm{GL}(2, F)$. The GL( $2, F)$-orbit of a regular semisimple element $Y$ contains an element of the form

$$
\left[\begin{array}{cc}
0 & B \\
C & 0
\end{array}\right] \quad \text { with } \operatorname{val}(B) \leq \operatorname{val}(C) \leq \operatorname{val}(B)+1
$$

We take $Y$ to have this anti-diagonal form. Here, $Y$ is not topologically nilpotent when $\operatorname{val}(C) \leq 0$. It thus suffices to prove $\mathrm{FT}\left(1_{\mathfrak{g}_{0}}\right)(Y)=0$ in this situation. For $X=\left[\begin{array}{cc}a & b \\ c & -a\end{array}\right]$, we have $\operatorname{trace}(X Y)=(b C+c B)$, and so

$$
\int_{\mathcal{T}_{\ell}} \psi(\operatorname{trace}(X Y)) d X=\int_{\mathcal{T}_{\ell}} \psi(b C+c B) d X
$$


CASE $\operatorname{val}(C)=\operatorname{val}(B)$ : We remark in this situation, $Y$ is either split ore elliptic unramified. We show the integral vanishes if $B, C \notin \wp$. Our strategy is to partition $\mathcal{T}_{\ell}$ into regions were the integral is zero.

Subcase $a \in \mathcal{R}_{F}$ : The condition for $X$ to be in $\mathfrak{g}_{0}$ is $a^{2}+b c \in \mathcal{R}_{F}$, and so $b c \in \mathcal{R}_{F}$. We consider two subcases based on $b$.

- Subcase $b \in \mathcal{R}$. Here, dependent on the valuation val( $(b)$, the variable $c$ runs over an ideal between $\wp^{0}$ and $\wp^{-\ell}$. The assumption $B \notin \wp$ means $c \rightarrow \psi(c B)$ is a non-trivial character on its allowed ideal and therefore, for fixed $a$ and $b$, the integral over $c$ is zero. We deduce the integral over the region in $\mathcal{T}_{\ell}$ which satisfies $a, b \in \mathcal{R}$ is zero.

- Subcase $b=\varpi^{-k} u$ with (integral) $k>0$ and $u$ a unit. The condition $b c \in \mathcal{R}$, is $c \in \wp^{k} \subset \wp$. If $\operatorname{val}(B) \leq-k$, then $c \rightarrow \psi(c B)$ is a non-trivial character; so integration of $c$ over $\wp^{k}$ is zero. If $-k<\operatorname{val}(B)$, then $\psi(c B)=1$; so $\psi(b C+c B)=$ $\psi(b C)$. Integration over $c \in \wp^{k}$ yields $\psi(b C)$ meas $\left(\wp^{k}\right)$. We note that $x \rightarrow \psi(x C)$ is a non-trivial character on $\mathcal{R}$, and $\psi((b+x) C)=\psi(b C) \psi(x C)$. If we integrate over all $b \in \wp^{-k} \backslash \wp^{-k+1}$ we get zero. We deduce the integral over the region in $\mathcal{T}_{\ell}$ which satisfies $a \in \mathcal{R}$ and $b \notin \mathcal{R}$ is zero.

CASE $a \notin \mathcal{R}_{F}$ : Write $a$ as $a=\varpi^{-k} u$ with $u$ a unit, and $k$ a positive integer (note $k \leq \ell$ to satisfy $\left.a \in \wp^{-\ell}\right)$. The condition $a^{2}+b c \in \mathcal{R}$ for $X$ to be in $\mathfrak{g}_{0}$ is thus $-b c \in \varpi^{-2 k} u^{2}+\mathcal{R}$. In particular, $b$ and $c$ are non-zero. Write $b$, as $b=\varpi^{\beta} v_{b}$, with $v_{b}$ a unit. The condition, $b, c \in \wp^{-\ell}$ means $-\ell \leq \beta \leq \ell-2 k$, and similarly for the valuation $\gamma=\operatorname{val}(c)$ of $c$.

- If $\beta \neq \gamma$, then by the symmetry of $b$ and $c$, we assume $\beta<-k<\gamma$. This imposes the condition $c \in-\varpi^{\gamma} v_{b}^{-1} u^{2}+\wp^{-\beta}$.

- If $\operatorname{val}(B)-\beta \leq 0$, then for fixed $a$ and $b$, the integral over $c$ is zero.

- If $\operatorname{val}(B)-\beta>0$, then $\psi(c B)=\psi\left(-\varpi^{\gamma} v_{b}^{-1} u^{2} B\right)$ is independent of $c \in-\varpi^{\gamma} v_{b}^{-1} u^{2}+$ $\wp^{-\beta}$. Thus, if we fix $a$ and $b$, and integrate $\psi(b C+c B)$ over $c$, we get

$$
\psi(b C) \psi\left(-\varpi^{\gamma} v_{b}^{-1} u^{2} B\right) \operatorname{meas}\left(\varpi^{(-2 k-\beta)}+\wp^{-\beta}\right) .
$$

If we perturb $b$ by $v_{b} x \in \wp^{-\operatorname{val}(B)}$ to $b^{\prime}=b+v_{b} x$, so $v_{b^{\prime}}=v_{b}\left(1+\varpi^{-\beta} x\right)$, then the corresponding $c^{\prime}$ satisfies $c^{\prime} \in-\varpi^{\gamma} v_{b}^{-1}\left(1+\varpi^{-\beta} x\right)^{-1} u^{2}+\wp^{-\beta}=-\varpi^{\gamma} v_{b}^{-1} u^{2}+$ $\varpi^{\gamma-\beta} v_{b}^{-1} x u^{2}+\wp^{-\beta}$. We deduce $\psi\left(c^{\prime} B\right)=\psi\left(-\varpi^{\gamma} v_{b}^{-1} u^{2} B\right)$ is independent of $x$. So, $\psi\left(b^{\prime} C+c^{\prime} B\right)=\psi(b C) \psi(x C) \psi\left(-\varpi^{\gamma} v_{b}^{-1} u^{2} B\right)$, and therefore, if we restrict to $b \in \varpi^{-\beta} \mathcal{R}^{\times}$integrate over $c^{\prime}$ followed by integration over $b$, we get zero.

- If $\beta=\gamma=-k$, we have

$$
X=\varpi^{-k}\left[\begin{array}{cc}
u & v_{b} \\
v_{c} & -u
\end{array}\right] \quad \text { with } u, v_{b}, v_{c} \text { units, }
$$

and the condition for $X \in \mathfrak{g}_{0}$ is $u^{2}+v_{b} v_{c} \in \wp^{2 k}$. We see the product $-v_{b} v_{c}$ must be a square in $\mathcal{R}^{\times}$. Conversely, if $-v_{b} v_{c}$ is a square, the condition on $u$ is $u^{2} \in-v_{b} v_{c}+\wp^{2 k}$. We fix $v_{c}$ and multiplicatively perturb $v_{b}$ by $1+\wp$, the integral of $\psi\left(\varpi^{-k}\left(v_{c} B+v_{b^{\prime}} C\right)\right)$ over $b^{\prime} \in \varpi^{-k} v_{b}(1+\wp)$, and $a=\varpi^{-k} u$ with $u^{2} \in-v_{b^{\prime}} v_{c}+\wp^{2 k}$. The integration over $a$ yields a constant independent of $b^{\prime}$, and then the integration over $b^{\prime}$ is zero. So the 
integral of $\psi\left(\varpi^{-k}\left(v_{c} B+v_{b^{\prime}} C\right)\right)$ over the region satisfying $X \in \mathfrak{g}_{0}$ and $a, b, c \in \varpi^{-k} \mathcal{R}^{\times}$ is zero.

CASE $\operatorname{val}(C)=\operatorname{val}(B)+1$ : The proof here is a minor modification of the case $\operatorname{val}(C)=$ $\operatorname{val}(B)$. We omit the details.

This completes the proof that $\mathrm{FT}\left(1_{\mathfrak{g}_{0}}\right)$ has support in $\mathfrak{g}_{\frac{1}{2}}$.

The statement about the support of $\mathrm{FT}\left(1_{\mathfrak{g}_{-k}}\right)$ for $k \geq 0$ follows from the elementary property $\mathfrak{g}_{k-1}=\varpi^{-1} \mathfrak{g}_{k}$, and elementary homogeneities of the Fourier transform.

\section{REFERENCES}

[B] Bernstein, J., Notes of lectures on Representations of p-adic Groups, Harvard University, Fall 1992, written by K. E. Rumelhart.

[BD] Bernstein, J., rédigé par Deligne, P., Le "centre" de Bernstein, "Représentations des Groupes Réductifs sur un Corps Local" written by J.-N. Bernstein, P. Deligne, D. Kazhdan and M.-F. Vignéras, Hermann, Paris, 1984.

[BKV] Bezrukavnikov, R., Kazhdan, D., Varshavsky, V., On the depth r Bernstein projector, arXiv:1504.01353v1, 36 pages.

[D] Dat, J.-F., Quelques propriétés des idempotents centraux des groupes p-adiques. (French) [Some properties of the central idempotents of p-adic groups] J. Reine Angew. Math. 554 (2003), pp. 69-103.

[IR] Ireland, K., Rosen, M., A classical introduction to modern number theory. Second edition. Graduate Texts in Mathematics, 84. Springer-Verlag, New York, 1990.

[HC] Harish-Chandra, Harmonic analysis on reductive p-adic groups. Notes by G. van Dijk. Lecture Notes in Mathematics, Vol. 162. Springer-Verlag, Berlin-New York, 1970

[Ka] Kim, J.-L., Dual blobs and Plancherel formulas. Bull. Soc. Math. France 132 (2004), no. 1, 55-80.

[Kb] Kim, J.-L., Supercuspidal representations: an exhaustion theorem. J. Amer. Math. Soc. 20 (2007), no. 2, 273-320 (electronic).

[MPa] Moy, A., Prasad, G., Unrefined minimal K-types for p-adic groups. Invent. Math. 116 (1994), no. 1-3, $393-408$.

[MPb] Moy, A., Prasad, G., Jacquet functors and unrefined minimal K-types. Comment. Math. Helv. 71 (1996), no. $1,98-121$.

[MTa] Moy, A., Tadić, M., The Bernstein center in terms of invariant locally integrable functions. Represent. Theory 6 (2002), pp. 313-329 (electronic).

[MTb] Moy, A., Tadić, M., Erratum to: "The Bernstein center in terms of invariant locally integrable functions" Represent. Theory 6 (2002), 313-329 (electronic). Represent. Theory 9 (2005), 455-456 (electronic).

[SS] Sally, P. J., Jr., Shalika, J. A., Characters of the discrete series of representations of SL(2) over a local field. Proc. Nat. Acad. Sci. U.S.A. 61 (1968), pp. 1231-1237.

Department of Mathematics, The Hong Kong University of Science and Technology, Clear Water Bay, Hong Kong, Email:amoy@ust.hk

Department of Mathematics, University of Utah, Salt Lake City, UT 84112 